\title{
WestVirginiaUniversity
}

THE RESEARCH REPOSITORY @ WVU

Graduate Theses, Dissertations, and Problem Reports

2004

\section{On flows of graphs}

Rui Xu

West Virginia University

Follow this and additional works at: https://researchrepository.wvu.edu/etd

\section{Recommended Citation}

Xu, Rui, "On flows of graphs" (2004). Graduate Theses, Dissertations, and Problem Reports. 2096.

https://researchrepository.wvu.edu/etd/2096

This Dissertation is protected by copyright and/or related rights. It has been brought to you by the The Research Repository @ WVU with permission from the rights-holder(s). You are free to use this Dissertation in any way that is permitted by the copyright and related rights legislation that applies to your use. For other uses you must obtain permission from the rights-holder(s) directly, unless additional rights are indicated by a Creative Commons license in the record and/ or on the work itself. This Dissertation has been accepted for inclusion in WVU Graduate Theses, Dissertations, and Problem Reports collection by an authorized administrator of The Research Repository @ WVU.

For more information, please contact researchrepository@mail.wvu.edu. 


\title{
On Flows of Graphs
}

\author{
Rui Xu \\ Dissertation submitted to the \\ Eberly College of Arts and Sciences \\ at West Virginia University \\ in partial fulfillment of the requirements \\ for the degree of \\ Doctor of Philosophy \\ in \\ Mathematics \\ Cun-Quan Zhang, Ph.D., Chair \\ Elaine M. Eschen, Ph.D. \\ John L. Goldwasser, Ph.D. \\ Hong-Jian Lai, Ph.D. \\ Jerzy Wojciechowski, Ph.D. \\ Department of Mathematics \\ Morgantown, West Virginia \\ 2004
}

Keywords: Graph, Integer Flow, Circular Flow, Bidirected Flow Copyright 2004 Rui Xu 


\title{
On Flows of Graphs
}

\author{
Rui Xu
}

\begin{abstract}
Tutte's 3-flow Conjecture, 4-flow Conjecture, and 5-flow Conjecture are among the most fascinating problems in graph theory. In this dissertation, we mainly focus on the nowherezero integer flow of graphs, the circular flow of graphs and the bidirected flow of graphs. We confirm Tutte's 3-flow Conjecture for the family of squares of graphs and the family of triangularly connected graphs. In fact, we obtain much stronger results on this conjecture in terms of group connectivity and get the complete characterization of such graphs in those families which do not admit nowhere-zero 3-flows. For the circular flows of graphs, we establish some sufficient conditions for a graph to have circular flow index less than 4, which generalize a new known result to a large family of graphs. For the Bidirected Flow Conjecture, we prove it to be true for 6-edge connected graphs.
\end{abstract}




\section{Dedication}

This dissertation is dedicated to my daughter, my wife, my parents and my whole family for their love, understanding, encouragement and support. 


\section{Acknowledgments}

I sincerely express my appreciation to my thesis advisor, Dr. Cun-Quan Zhang, for his excellent guidance, advice and continual support and encouragement. It has been my great pleasure to work under his supervision. Without him, this piece of work would have never come to completion.

I would like to thank the other committee members: Dr. Elaine M. Eschen, Dr. John L. Goldwasser, Dr. Hong-Jian Lai and Dr. Jerzy Wojciechowski for their support and help during my study here .

I would also like to thank the Department of Mathematics at West Virginia University for providing me with an excellent study environment during my four years study as a graduate student. My thanks also goes to all the professors who have given me support and help in my study and in my daily life. 


\section{Contents}

1 Introduction $\quad 1$

2 Nowhere-zero 3-flows in squares of graphs 4

2.1 Introduction . . . . . . . . . . . . . . . . . . . 4

2.2 Splitting operation, flow extension and lemmas . . . . . . . . . 8

2.3 Proof of the main theorem . . . . . . . . . . . . . . . . . . 12

$3 \quad$ Group connectivity of triangularly connected graphs $\quad 17$

3.1 Introduction . . . . . . . . . . . . . . . . . . 17

3.2 Lemmas . . . . . . . . . . . . . . . . . . . . . . . . . 21

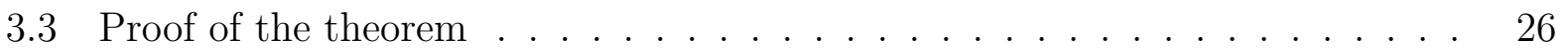

4 On circular flows of graphs $\quad 30$

4.1 Introduction . . . . . . . . . . . . . . . . . . . 30

4.2 Lemmas . . . . . . . . . . . . . . . . . . . . . . 32

4.3 Main results . . . . . . . . . . . . . . . . . . . . . 33 
5 On flows of bidirected graphs

5.1 Introduction . . . . . . . . . . . . . . . . . . . 36

5.2 Proof of Proposition 5.1 .4 and its applications . . . . . . . . . . . . . . 39

5.3 Proof of Theorem 5.1.5 . . . . . . . . . . . . . . . . . 41

5.4 Proof of Theorem 5.1.6 . . . . . . . . . . . . . . . . . . 44

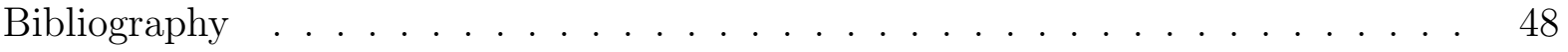




\section{Chapter 1}

\section{Introduction}

The map coloring problem is considered one of the major catalysts of the tremendous development of graph theory in its 260-year history. It is, thus, not surprising that graph theory coloring and its related problems have always been in the main line of graph theory research. It was observed by Tutte that the problem of the face-coloring of an embedded (planar) graph can be formulated in terms of integer flows in the graph. Since then, the topic of integer flow has been one of the most attractive in graph theory.

Tutte had three famous fascinating flow conjectures: 3-flow conjecture, 4-flow conjecture and 5-flow conjecture. There are a lot of partial results for these three conjectures (see the survey papers by Jackson [22], Jaeger [25] and Younger [52] and the book by Zhang [53]). All three of these conjectures are still open.

The concept of circular flow, introduced by Goddyn, Tarsi and Zhang in [16], is a generalization of integer flows. With the introduction of circular flow, the concept of integer flow was successfully extended to real values. By the relation between the circular flow

index and the flow index, circular flows can be considered as a refinement of integer flows. Therefore, for each integer flow conjecture, there is a corresponding circular flow conjecture. 
The concept of bidirected flow, introduced by Bouchet [7], is another generalization of integer flows. It is also motivated by the relation between the flow and the tension. For a graph drawn on an orientable surface of higher genus, flows are not dual to colorings, but to local-tensions. By Seymour's theorem, every graph on an orientable surface without the obvious obstruction has a nowhere-zero 6-local-tension. Bouchet conjectured that the same holds true on non-orientable surfaces and conjectured that every bidirected graph without the obvious obstruction should have a nowhere-zero bidirected 6-flow. This is known as the bidirected 6-flow conjecture.

In this dissertation, we mainly focus on the 3-flow conjecture, the circular flow problem and the bidirected flow conjecture.

In Chapter 2, we study the 3-flows of a family of graphs: squares of graphs. Squares of graphs can be used to model atom-bond networks with bond-angle forces, which arise in the study of glasses. Also, the problem of coloring squares of graphs has been studied recently for its applications to frequency allocation. Many properties have been studied for the family of squares of graphs by other authors, for example, the coloring number [1], the edge clique cover number [27], the existence of factors [37, 2] and the Hamiltonian property [13, 40]. Here, we prove that the Tutte's 3-flow conjecture is true for this family of graphs. In fact, we give a complete characterization of those graphs in this family which admit nowhere-zero 3-flows.

In Chapter 3, we study another family of graphs: triangularly connected graphs. The motivation of this work is the weak 3 -flow conjecture, which states that the 3 -flow conjecture is true for graphs with each edge contained in a triangle. Note that these graphs can be obtained by attaching together triangularly connected graphs. Therefore, if we can get the structure result about nowhere-zero 3-flows of triangularly connected graphs, it will help to completely solve this weak flow conjecture. Here, we prove that Tutte's 3 -flow conjecture is true for this family of graphs. In fact, we give a complete characterization of those graphs in this family which admit nowhere-zero 3-flows. Furthermore, we get more general results 
in terms of $Z_{3}$-connectivity for this family of graphs.

In Chapter 4, we get some sufficient conditions for graphs with circular flow index less than 4. As an immediate corollary, we give a simple proof of a result obtained recently by Galluccio and Goddyn (Combinatorica, 2002), and obtain a larger family of such graphs.

In Chapter 5, we focus on the bidirected flows of graphs and prove that Bouchet's bidirected 6-flow conjecture is true for 6-edge connected graphs. 


\section{Chapter 2}

\section{Nowhere-zero 3-flows in squares of graphs}

It was conjectured by Tutte [6] that every 4-edge connected graph admits a nowhere-zero 3-flow. In this paper, we give a complete characterization of graphs whose squares admit nowhere-zero 3-flows. As a corollary, we confirm Tutte's 3-flow conjecture for the family of squares of graphs.

\section{$2.1 \quad$ Introduction}

All graphs considered in this chapter are finite and simple. Let $G=(V, E)$ be a graph with vertex set $V$ and edge set $E$. For any $v \in V(G)$, we use $d_{G}(v), N_{G}(v)$ to denote the degree and the neighbor set of $v$ in $G$, respectively. The minimal degree of a vertex of $G$ is denoted by $\delta(G)$. We use $K_{m}$ for a complete graph on $m$ vertices, $P_{t}$ for a path of length $t$ and $W_{4}$ for a graph obtained from a 4-circuit by adding a new vertex $x$ and edges joining $x$ to all the vertices on the circuit. We call $x$ the center of this $W_{4}$ and each edge with $x$ as one endpoint is called a center edge. Let $D$ be an orientation of $G$. Then the set of all edges with tails 
(or heads) at a vertex $v$ is denoted by $E^{+}(v)$ (or $E^{-}(v)$ ). If an edge $u v$ is oriented from $u$ to $v$ under $D$, then we say $D(u v)=u \rightarrow v$. The square of $G$, denoted by $G^{2}$, is the graph obtained from $G$ by adding all the edges that join distance 2 vertices in $G$. We refer the reader to [6] for terminology not defined in this chapter.

Definition 2.1.1 Let $D$ be an orientation of $G$ and $f$ be a function: $E(G) \mapsto Z$. Then

(1). The ordered pair $(D, f)$ is called a $k$-flow of $G$ if $-k+1 \leq f(e) \leq k-1$ for every edge $e \in E(G)$ and $\sum_{e \in E^{+}(v)} f(e)=\sum_{e \in E^{-}(v)} f(e)$ for every $v \in V(G)$.

(2). The ordered pair $(D, f)$ is called a Modular $k$-flow of $G$ if for every $v \in V(G)$, $\sum_{e \in E^{+}(v)} f(e) \equiv \sum_{e \in E^{-}(v)} f(e)(\bmod k)$.

The support of a k-flow (modular $k$-flow) $(D, f)$ of $G$ is the set of edges of $G$ with $f(e) \neq 0(f(e) \not \equiv 0(\bmod k))$, and is denoted by $\operatorname{supp}(f) . A k$-flow $(D, f)$ (Modular $k$-flow) of $G$ is nowhere-zero if $\operatorname{supp}(f)=E(G)$.

Definition 2.1.2 Let $\Gamma$ be an Abelian group ( an additive group with " 0 " as the identity). $A$ C-flow of $G$ is a flow $(D, f)$ where $f$ is a function: $E(G) \mapsto \Gamma$.

For convenience, a nowhere-zero $k$-flow $(\Gamma$-flow) is abbreviated as a $k$-NZF $(\Gamma-\mathbf{N Z F})$. The concept of integer-flow was introduced by Tutte $([45,46]$, also see $[52,25])$ as a refinement and generalization of face-coloring and edge-3-coloring problems. The following three conjectures are the most well-known open problems in this subject.

The 5-flow Conjecture (Tutte [46]) Every 2-edge connected graph admits a 5-NZF.

The 4-flow Conjecture (Tutte [48]) Every 2-edge connected graph containing no subdivision of the Petersen graph admits a $4-\mathrm{NZF}$.

The 3-flow Conjecture (Tutte, unsolved problem 48 in [6]) Every 4-edge connected graph admits a $3-\mathrm{NZF}$. 
There are many partial results on these three conjectures, but all of them are still open. For the $k$-NZF and $\Gamma-\mathrm{NZF}$, Tutte obtained the following result.

Theorem 2.1.3 (Tutte [46]) Let $\Gamma$ be an Abelian group of order $k$. A graph $G$ admits a $k-N Z F$ if and only if $G$ admits a $\Gamma-N Z F$.

For the 5-flow conjecture, Jaeger and Kilpatrick obtained the following result independently.

Theorem 2.1.4 (Jaeger [23], Kilpatrick [29]) Every 2-edge connected graph admits a 8-NZF.

The strongest partial result for the 5-flow conjecture is due to Seymour.

Theorem 2.1.5 (Seymour [41]) Every 2-edge connected graph admits a 6-NZF.

The 4-flow conjecture has been confirmed for planar graphs. The result is also known as the Four Color Theorem.

Theorem 2.1.6 (Appel and Haken $[3,4,5]$ ) The 4-flow conjecture is true for planar graphs.

For the 3-flow conjecture, Jaeger obtained the best approach and Grötzsch proved it to be true for planar graphs.

Theorem 2.1.7 (Jaeger [23]) Every 4-edge connected graph admits a 4-NZF.

Theorem 2.1.8 (Grötzsch [17]) Every 4-edge connected planar graph is face-3-colorable, equivalently, admits a $3-N Z F$. 
Jaeger proposed the following weaker version of the 3-flow conjecture.

The Weak 3-flow Conjecture (Jaeger [25]) There is an integer $k$ such that every $k$-edge connected graph admits a $3-\mathrm{NZF}$.

The following is one approach to this conjecture.

Theorem 2.1.9 (Lai and Zhang [31]) Let $G$ be a k-edge connected graph with $t$ odd vertices. If $k \geq 4\left\lceil\log _{2}^{t}\right\rceil$, then $G$ admits a $3-N Z F$.

In this chapter, squares of graphs admitting 3-NZF's will be characterized. The following families of graphs are the exceptions in the main theorem.

Definition 2.1.10 $\mathcal{T}_{1,3}=\left\{T \mid T\right.$ is a tree and $d_{T}(v)=1$ or 3 for every $\left.v \in V(T)\right\}$

Definition 2.1.11 $\overline{\mathcal{T}}_{1,3}=\left\{T \mid T \in \mathcal{T}_{1,3}\right.$ or $T$ is a 4-circuit or $T$ can be obtained from some $T^{\prime} \in \mathcal{T}_{1,3}$ by adding some edges each of which joins a pair of distance 2 leaves of $\left.T^{\prime}\right\}$

The following is the main result of this chapter.

Theorem 2.1.12 Let $G$ be a connected simple graph. Then $G^{2}$ admits a 3-NZF if and only if $G \notin \overline{\mathcal{T}}_{1,3}$.

An immediate corollary of Theorem 2.1.12 is the following partial result for Tutte's 3-flow conjecture.

Corollary 2.1.13 Let $G$ be a graph. If $\delta\left(G^{2}\right) \geq 4$ then $G^{2}$ admits a $3-N Z F$.

This research is motivated by the 3 -flow conjecture and the following open problem: 
Conjecture 2.1.14 (Zhang [54]) If every edge of a 4-edge connected graph $G$ is contained in a 3 -circuit, then $G$ admits a $3-N Z F$.

Theorem 2.1.12 and the following early results are partial results for Conjecture 2.1.14.

Theorem 2.1.15 (Catlin [9]) If every edge of a graph $G$ is contained in a circuit of length at most 4 , then $G$ admits a $4-N Z F$.

Theorem 2.1.16 (Lai [32]) Every 2-edge connected, locally 3-edge connected graph admits a $3-N Z F$.

Theorem 2.1.17 (Imrich and Skrekovski [20]) Let $G$ and $H$ be two graphs. Then $G \times H$ admits a 3-NZF if both $G$ and $H$ are bipartite.

\subsection{Splitting operation, flow extension and lemmas}

Definition 2.2.1 (A special splitting operation) Let $G$ be a graph and $e=x y \in E(G)$. The graph $G_{* e}$ is obtained from $G$ by deleting the edge $e$ and adding two new vertices $x^{\prime}$ and $y^{\prime}$ and adding two new edges, $e_{x}$ and $e_{y}$, joining $x$ and $y^{\prime}, y$ and $x^{\prime}$, respectively.

Definition 2.2.2 Let $G$ be a graph, let $(D, f)$ be a 3-flow of $G$ and let $F \subseteq E(G) \backslash \operatorname{supp}(f)$. A 3-flow $\left(D^{\prime}, f^{\prime}\right)$ of $G$ is called an $(F, f)$-changer if $F \cup \operatorname{supp}(f) \subseteq \operatorname{supp}\left(f^{\prime}\right)$.

Lemma 2.2.3 ([45]) A graph $G$ admits a k-flow $\left(D, f_{1}\right)$ if and only if $G$ admits a Modular $k$-flow $\left(D, f_{2}\right)$ such that $f_{1}(e) \equiv f_{2}(e)(\bmod k)$ for each $e \in E(G)$.

An orientation of a graph $G$ is called a modular 3-orientation if $\left|E^{+}(v)\right| \equiv\left|E^{-}(v)\right|(\bmod 3)$, for every $v \in V(G)$. The following result appears in $[25,43,52]$, but by Lemma 2.2.3, we can attribute it to Tutte. 
Lemma 2.2.4 ([45]) Let $G$ be a graph. Then $G$ admits a 3-NZF if and only if $G$ has a modular 3-orientation.

A partial 3-orientation $D$ of $G$ is an orientation of some edges of $G$ satisfying $\left|E^{+}(v)\right| \equiv$ $\left|E^{-}(v)\right|(\bmod 3)$, for any $v \in V(G)$. The support of $D$ is the set of edges oriented under $D$ and is denoted by $\operatorname{supp}(D)$. Clearly the partial orientation obtained by reversing every oriented edge of a partial 3-orientation is also a partial 3-orientation.

Let $D$ be a partial 3-orientation of $G$ and let $C=v_{0} v_{1} \cdots v_{k-1} v_{0}$ be a circuit of $G$. A circuit-operation along $C$ is defined as following: For $0 \leq i \leq k-1$, if $D\left(v_{i} v_{i+1}\right)=v_{i} \rightarrow$ $v_{i+1}(\bmod k)$, then reverse the direction of this edge; if $\left(v_{i} v_{i+1}\right)(\bmod k)$ is not oriented under $D$, then orient it as $v_{i} \rightarrow v_{i+1}$; if $D\left(v_{i} v_{i+1}\right)=v_{i+1} \rightarrow v_{i}(\bmod k)$ then $v_{i} v_{i+1}$ loses it's orientation.

Lemma 2.2.5 Let $G$ be a graph, $(D, f)$ be a 3-flow of $G$ and $H$ be a subgraph of $G$

(1). If $H \cong W_{4}$ and $e \in E(H) \backslash \operatorname{supp}(f)$ is a center edge, then an $(\{e\}, f)$-changer exists.

(2). If $H$ is a circuit of length 3 with $E(H) \cap \operatorname{supp}(f)=\{e\}$, then an $(E(H) \backslash\{e\}, f)$ changer exists.

Proof. (1). Since $H \cong W_{4}$, let $x$ be the center of $H$ and let $u_{1} u_{2} u_{3} u_{4} u_{1}$ be the 4-circuit $H \backslash x$. Since $G$ has a 3-flow $(D, f)$, then $G$ has a partial 3-orientation $D^{*}$ with $\operatorname{supp}\left(D^{*}\right)=\operatorname{supp}(f)$. We need only to find a partial 3-orientation $D^{\prime}$ such that $\operatorname{supp}\left(D^{*}\right) \cup\{e\} \subseteq \operatorname{supp}\left(D^{\prime}\right)$. Since $e$ is a center edge, without loss of generality, assume that $e=x u_{1}$.

First we assume $E(H) \backslash\{e\} \subseteq \operatorname{supp}\left(D^{*}\right)$. Without loss of generality, assume $D^{*}\left(u_{1} u_{2}\right)=$ $u_{1} \rightarrow u_{2}$. Then $D^{*}\left(u_{2} x\right)=x \rightarrow u_{2}$. Otherwise, we do a circuit-operation along $u_{1} u_{2} x u_{1}$ and then get a needed partial 3-orientation $D^{\prime}$ of $G$. For the same reason, $u_{4}$ must be the tail (or head) of both $u_{1} u_{4}$ and $x u_{4}$. By symmetry, we consider the following two cases. 
Case 1. $D^{*}\left(u_{1} u_{4}\right)=u_{1} \rightarrow u_{4}$ and $D^{*}\left(x u_{4}\right)=x \rightarrow u_{4}$.

We may assume that $u_{3}$ is the tail (or head) of all edges incident with it in $H$. Otherwise, there exists a directed 2-path $x u_{3} u_{i}$ (or $u_{i} u_{3} x$ ) for some $i \in\{2,4\}$. Then we do circuitoperations along $x u_{3} u_{i} x$ (or $u_{i} u_{3} x u_{i}$ ) and along $u_{1} u_{i} x u_{1}$. Therefore, we get a needed partial 3-orientation of $D^{\prime}$ of $G$.

If all edges in $H$ have $u_{3}$ as a tail, then we do circuit-operations along $x u_{1} u_{4} x$, along $u_{4} x u_{3} u_{4}$, along $x u_{3} u_{2} x$ and along $u_{2} x u_{1} u_{2}$; If all edges in $H$ have $u_{3}$ as a head, then we do circuit-operations along $u_{1} u_{2} u_{3} x u_{1}$ and along $u_{3} x u_{4} u_{3}$. In both cases, we get a needed partial 3-orientation $D^{\prime}$ of $G$.

Case 2. $D^{*}\left(u_{1} u_{4}\right)=u_{4} \rightarrow u_{1}$ and $D^{*}\left(x u_{4}\right)=u_{4} \rightarrow x$.

Similar to Case 1, we may assume $u_{3}$ be the tail (or head) of all edges incident with it in $H$. If all edges in $H$ have $u_{3}$ as a tail, then we do circuit-operations along $x u_{1} u_{4} x$, along $u_{3} u_{4} u_{1} u_{2} u_{3}$ and along $u_{3} x u_{2} u_{3}$; If all edges in $H$ have $u_{3}$ as a head, then we do circuitoperations along $u_{1} x u_{2} u_{1}$, along $u_{4} u_{1} u_{2} u_{3} u_{4}$ and along $u_{4} x u_{3} u_{4}$. In both cases, we get a needed partial 3-orientation $D^{\prime}$ of $G$.

If $\operatorname{supp}\left(D^{*}\right)$ misses some other edges of $E(H)$, say $e^{*}=a b \in E(H) \backslash \operatorname{supp}\left(D^{*}\right)$, then we define $D^{*}(a b)=a \rightarrow b$ or $b \rightarrow a$, by the proof of Case 1 and Case 2, we can find a needed $D^{\prime}$ of $G$.

(2). it is trivial.

Lemma 2.2.6 For each $G \in \overline{\mathcal{T}}_{1,3}$ and each $e_{0} \in E(G)$, the graph $G^{2}$ admits a 3-flow $(D, f)$ such that $\operatorname{supp}(f)=E\left(G^{2}\right) \backslash\left\{e_{0}\right\}$

Proof. Induction on $|E(G)|$. It is obviously true for graphs $G$ with $G^{2}=K_{4}$ (including $G=C_{4}$, the circuit of length 4). So, assume that $|V(G)| \geq 5$ and let $D$ be any fixed orientation of $G^{2}$. 
Let $e=x y$ with $d_{G}(x)=d_{G}(y)=3$. Then $G_{* e}$ consists of two components, say $G_{1}$ and $G_{2}$. Clearly, $G_{1}, G_{2} \in \overline{\mathcal{T}}_{1,3}$. Without loss of generality, let $e_{0} \in E\left(G_{1}\right)$. By induction, $G_{1}^{2}$ admits a 3 -flow $\left(D, f_{1}\right)$ such that $\operatorname{supp}\left(f_{1}\right)=E\left(G_{1}^{2}\right) \backslash\left\{e_{0}\right\}$ and $G_{2}^{2}$ admits a 3 -flow $\left(D, f_{2}\right)$ that $\operatorname{supp}\left(f_{2}\right)=E\left(G_{2}^{2}\right) \backslash\{e\}$.

Then, identifying the split vertices and edges, back to $G,\left(D, f_{1}+f_{2}\right)$ is a 3 -flow $(D, f)$ with $\operatorname{supp}(f)=E\left(G^{2}\right) \backslash\left\{e_{0}\right\}$.

Lemma 2.2.7 (1). Let $G$ be a k-path with $k \geq 2$ or an $m$-circuit with $m=3$ or $m \geq 5$. Then $G^{2}$ admits a $3-N Z F$.

(2). Let $G$ be a graph obtained from an $r$-circuit $x_{0} x_{1} \cdots x_{r-1} x_{0}$ by attaching an edge $x_{i} v_{i}$ at each $x_{i}$ for $0 \leq i \leq r-1$, where $v_{i} \neq v_{j}$ if $i \neq j$. Then $G^{2}$ admits a $3-N Z F$.

(3). Let $G$ be a graph obtained from an $m$-circuit $x_{0} x_{1} \cdots x_{m-1} x_{0}$ by attaching an edge $x_{m-1} v$ at $x_{m-1}$ alone, where $m \geq 5$. Then $G^{2}$ admits a $3-N Z F$.

Proof. (1). If $G$ is an $m$-circuit with $m=3$ or $m \geq 5$, then $G^{2}$ is a cycle (every vertex is of even degree) and $G^{2}$ admits 2-NZF. If $G$ is a $k$-path with $k \geq 2$, by induction on $k$ and using Lemma 2.2.5-(2), $G^{2}$ admits a 3-NZF.

(2). For $r \geq 5$ (or $r=3$ ): let $D$ be an orientation such that $v_{i}(0 \leq i \leq r-1)$ is the tail of every edge of $G^{2}$ incident with it and all the other edges are oriented as $x_{i} \rightarrow x_{i+1}, x_{i} \rightarrow x_{i+2}$ $(\bmod r)\left(\right.$ or $x_{i} \rightarrow x_{i+1}(\bmod 3)$ only for $\left.r=3\right)$. Obviously, $D$ is a modular 3-orientation of $G^{2}$.

For $r=4$ : let $D$ be the orientation such that $v_{0}$ and $v_{2}$ be the tail of every edge of $G^{2}$ incident with it, $v_{1}$ and $v_{3}$ be the head of every edge of $G^{2}$ incident with it, $x_{0} x_{1} x_{3} x_{2} x_{0}$ as a directed circuit and other edges are oriented as $x_{3} \rightarrow x_{0}, x_{1} \rightarrow x_{2}$. Obviously, $D$ is a modular 3-orientation of $G^{2}$. 
(3). Orient all the edges as $x_{i} \rightarrow x_{i+1}, x_{i} \rightarrow x_{i+2}(\bmod m)$ for $0 \leq i \leq m-1$ and let $v$ be the tail of every edge of $G^{2}$ incident with it. Then reverse the direction of the following edges: $x_{0} x_{m-1}, x_{0} x_{m-2}$. Clearly, this orientation is a modular 3-orientation of $G^{2}$.

\subsection{Proof of the main theorem}

Proof. $\Longrightarrow$ By contradiction. Suppose $G \in \overline{\mathcal{T}}_{1,3}$. Let $G$ be a counterexample with $|V(G)|+$ $|E(G)|$ as small as possible. Clearly $|V(G)| \geq 5$ and $G$ contains no circuits. So $G \in \mathcal{T}_{1,3}$. Let $v \in V(G)$ be a degree 3 vertex such that $N_{G}(v)=\left\{v_{1}, v_{2}, v_{3}\right\}, d_{G}\left(v_{1}\right)=d_{G}\left(v_{2}\right)=1$. Clearly, $G_{1}=G \backslash\left\{v_{1}, v_{2}\right\} \in \mathcal{T}_{1,3}$. Since $G^{2}$ has a modular 3-orientation $D$ and both $v_{1}$ and $v_{2}$ are degree 3 vertices in $G^{2}$, then this orientation restricted to the edge set of $G_{1}^{2}$ will generate a modular 3-orientation of $G_{1}^{2}$. Therefore, $G_{1}^{2}$ admits a 3-NZF, a contradiction.

$\Longleftarrow$ Let $G$ be a counterexample to the theorem such that

(i). $|E(G)|-|V(G)|$ is as small as possible,

(ii). subject to (i), $|E(G)|$ is as small as possible.

Note that $|E(G)|-|V(G)|+1$ is the rank of the cycle space of $G$.

Claim 1. Let $e_{0}=x y \in E(G)$. If $d_{G}(x) \geq 3$ and $d_{G}(y) \geq 2$, then $x y$ is not a cut edge of $G$.

If $e_{0}$ is a cut-edge, then at least one component of $G_{* e_{0}}$ is not in $\overline{\mathcal{T}}_{1,3}$, say, $G_{1}$ is not, while $G_{2}$ might be. By induction, let $\left(D, f_{i}\right)$ be a 3 -flow of $G_{i}^{2}$ for each $i=1,2$ such that $f_{1}$ is nowhere-zero, $f_{2}$ might miss only one edge $e_{x}$ (that is a copy of $e_{0}$ ). Without loss of generality, assume that $f_{1}\left(e_{y}\right)+f_{2}\left(e_{x}\right) \not \equiv 0(\bmod (3))$. Then, identifying the split vertices and edges, back to $G,\left(D, f_{1}+f_{2}\right)$ is a nowhere-zero Modular 3-flow of $G^{2}$. By Lemma 2.2.3, $G^{2}$ admits a 3 -NZF, a contradiction. 
Claim 2. $d_{G}(x) \leq 3$ for any $x \in V(G)$.

Otherwise, assume that $d_{G}(x) \geq 4$ for some vertex $x \in V(G)$. Clearly $G \not K_{1, m}$ for $m \geq 4$ since $K_{1, m}$ is not a counterexample. So there exists $e_{0}=x y \in E(G)$ with $d_{G}(y) \geq 2$. By Claim 1, $e_{0}$ is not a cut edge of $G$ and $G_{1}=G_{* e_{0}} \notin \overline{\mathcal{T}}_{1,3}$. Then by (i), $G_{1}^{2}$ admits a 3-NZF.

In $G_{1}^{2}$, identify $x$ and $x^{\prime}, y$ and $y^{\prime}$, and use one edge to replace two parallel edges, by Lemma 2.2.3, we will get $G^{2}$ and a Modular 3-flow $(D, f)$ of $G^{2}$ such that $E\left(G^{2}\right) \backslash \operatorname{supp}(f) \subseteq$ $\left\{x v\right.$ or $\left.y w \mid v \in N_{G}(y), w \in N_{G}(x)\right\}$. Let $C(x)=G^{2}\left[N_{G}(x) \cup\{x\}\right]$. Then $C(x)$ is a clique of order at least 5 . We are to adjust $(D, f)$ so that the resulting Modular 3-flow $\left(D, f^{\prime}\right)$ of $G^{2}$ misses only edges of $\{u v \mid u, v \in V(C(x))\}$. For each edge $x v$ which is missed by $\operatorname{supp}(f)$ and $x v \notin E(C(x))$, xyvx must be a circuit of $G^{2}$, so let $\left(D, f_{x v}\right)$ be a 3-flow of $G^{2}$ with $\operatorname{supp}\left(f_{x v}\right)=\{x y, y v, x v\}$ and $f_{x v}(y v)+f(y v) \not \equiv 0(\bmod 3)$. Now $\left(D, f+f_{x v}\right)$ is a Modular 3 -flow of $G^{2}$ whose support contains $x v, y v$, but may miss $x y$. Repeat this adjustment and do the similar adjustment for the edges yw not in the support until we get a Modular 3-flow $\left(D, f^{\prime}\right)$ of $G^{2}$ such that $E\left(G^{2}\right) \backslash \operatorname{supp}\left(f^{\prime}\right) \subseteq E(C(x))$. Since each edge in $C(x)$ is contained in some $K_{5}$ and thus is a center edge in some $W_{4}$, by Lemma 2.2 .3 and Lemma 2.2.5-(1), $G^{2}$ admits a $3-\mathrm{NZF}$, a contradiction.

Claim 3. No degree 2 vertex is contained in a 3-circuit.

By contradiction. Assume $x y z x$ is a circuit of $G$ with $d_{G}(x)=2$. If $d_{G}(y)=2$, then we must have $d_{G}(z)=3$. Therefore $G_{1}=G \backslash\{x y\} \notin \overline{\mathcal{T}}_{1,3}$ and $G_{1}^{2}=G^{2}$, contradicting (ii). So $d_{G}(y)=d_{G}(z)=3$.

Let $N_{G}(y)=\left\{x, y^{\prime}, z\right\}$ and $N_{G}(z)=\left\{x, y, z^{\prime}\right\}$. Let $G_{1}=G-\{x\}$. Since $\left(N_{G}(y) \cap N_{G}(z)\right) \backslash$ $\{x\}=\emptyset$ (otherwise, let $G_{2}=G \backslash\{y z\}$, then $G_{2}^{2}=G^{2}, G_{2} \notin \overline{\mathcal{T}}_{1,3}$, contradicting (ii)) and $d_{G_{1}}(y)=2$, then $G_{1} \notin \overline{\mathcal{T}}_{1,3}$. So $G_{1}^{2}$ admits a 3 -NZF. Since $E\left(G^{2}\right) \backslash E\left(G_{1}^{2}\right)=\left\{x y, x y^{\prime}, x z, x z^{\prime}\right\}$, by Lemma $2.2 .5-(2), G^{2}$ admits a $3-\mathrm{NZF}$, a contradiction. 
Claim 4. No degree 2 vertex of $G$ is contained in a 4-circuit.

Assume $C=x u_{1} u_{2} u_{3} x$ is a 4 -circuit of $G$ and $d_{G}(x)=2$. By Claim 3, $u_{1} u_{3} \notin E(G)$. Let $u_{i}^{\prime}$ be the adjacent vertex of $u_{i}$ which is not in $V(C)$ if $d_{G}\left(u_{i}\right)=3$ for some $i \in\{1,2,3\}$. Let $G_{1}=G \backslash\{x\}$. We consider the following 3 cases.

Case 1. $d_{G}\left(u_{1}\right)=d_{G}\left(u_{3}\right)=2$.

Then $d_{G}\left(u_{2}\right)=3$ and $d_{G}\left(u_{2}^{\prime}\right) \geq 2$ (if $d_{G}\left(u_{2}^{\prime}\right)=1$, it's easy to show $G^{2}$ admits a 3 -NZF). Clearly, $u_{2} u_{2}^{\prime}$ is a cut edge, contradicting Claim 1 .

Case 2. Exactly one of $u_{1}, u_{3}$ has degree 3.

Assume $d_{G}\left(u_{1}\right)=3$ and $d_{G}\left(u_{3}\right)=2$. Since $d_{G_{1}}\left(u_{1}\right)=2$, if $d_{G_{1}}\left(u_{1}^{\prime}\right)=2$ then $u_{1}^{\prime}$ is not contained in a 3-circuit in $G$ (by Claim 3), and so $G_{1} \notin \overline{\mathcal{T}}_{1,3}$. By induction, $G_{1}^{2}$ admits a 3-NZF. Since $E\left(G^{2}\right) \backslash E\left(G_{1}^{2}\right)=\left\{x u_{1}^{\prime}, x u_{1}, x u_{2}, x u_{3}\right\}$ and $G^{2}\left[V(C) \cup\left\{u_{1}^{\prime}\right\}\right]$ contains a $W_{4}$ with $x$ as its center, by Lemma 2.2.5-(1), $G^{2}$ admits a $3-\mathrm{NZF}$, a contradiction.

Case 3. $d_{G}\left(u_{1}\right)=d_{G}\left(u_{3}\right)=3$.

If $u_{1}^{\prime}=u_{3}^{\prime}$, then $u_{1}^{\prime} u_{1} u_{2} u_{3}$ is a 3 -path, otherwise $u_{1}^{\prime} u_{1} u_{2} u_{3} u_{3}^{\prime}$ is 4 -path. In both cases $G_{1}^{2}$ admits a 3 -NZF. Since $E\left(G^{2}\right) \backslash E\left(G_{1}^{2}\right)=\left\{x u_{1}^{\prime}, x u_{1}, x u_{2}, x u_{3}, x u_{3}^{\prime}\right\}$ and each edge $x u_{i}$ or $x u_{j}^{\prime}$ is contained in some $W_{4}$ in $G^{2}$ as a center edge for $1 \leq i \leq 3$ and $j=1,3$, by Lemma 2.2.5-(1), $G^{2}$ admits a 3 -NZF. a contradiction.

Claim 5. For any $v \in V(G), d_{G}(v) \neq 2$.

Otherwise, if there exists $v \in V(G)$ such that $d_{G}(v)=2$, then by Claim 3-4, $v$ is not contained in any circuits of length 3 or 4 . By Lemma 2.2.7-(1), $G$ cannot be a $k$-path with $k \geq 2$ or an $m$-circuit with $m=3$ or $m \geq 5$. Let us consider the following cases.

Case 1. There exists a path $P_{m}=v_{1} v_{2} \cdots v_{m}$ such that $m \geq 3, v=v_{t}$ for some $2 \leq t \leq m-1, d_{G}\left(v_{k}\right)=2$ for $2 \leq k \leq m-1$ and $d_{G}\left(v_{1}\right) \neq 2, d_{G}\left(v_{m}\right) \neq 2$. 
Clearly, at least one of $v_{1}, v_{m}$ has degree 3 . If $d_{G}\left(v_{i}\right)=3$ for $i=1$, or $m$, let $N_{G}\left(v_{i}\right) \backslash$ $V\left(P_{m}\right)=\left\{v_{i}^{\prime}, v_{i}^{\prime \prime}\right\}$. Clearly, $G_{1}=G \backslash\left\{v_{2}, v_{3}, \ldots, v_{m-1}\right\} \notin \overline{\mathcal{T}}_{1,3}$ (because by Claim 3, degree 2 vertices are not contained in any 3 -circuits of $G$ ). By Claim 1, $G_{1}$ is connected. So $G_{1}^{2}$ admits a 3 -NZF $\left(D, f_{1}\right)$. By Lemma 2.2.7-(1), $P_{m}^{2}$ admits a 3 -NZF $\left(D, f_{2}\right)$. Then $G^{2}$ admits a 3-flow $(D, f)$ with $\operatorname{supp}(f)=\operatorname{supp}\left(f_{1}\right) \cup \operatorname{supp}\left(f_{2}\right)$. By Claim 3-4, $E\left(G^{2}\right) \backslash \operatorname{supp}(f)=$ $\left\{v_{2} v_{1}^{\prime}, v_{2} v_{1}^{\prime \prime}, v_{m-1} v_{m}^{\prime}, v_{m-1} v_{m}^{\prime \prime}\right\}$, then by Lemma 2.2.5-(2), $G^{2}$ admits a $3-\mathrm{NZF}$, a contradiction.

Case 2. There exists a $m$-circuit $C=v_{1} v_{2} \cdots v_{m} v_{1}$ with $m \geq 5, d_{G}\left(v_{i}\right)=2$ for $1 \leq i \leq$ $m-1, d_{G}\left(v_{m}\right)=3$ and $v=v_{t}$ for some $1 \leq t \leq m-1$.

Suppose that $v_{0} \in N_{G}\left(v_{m}\right) \backslash V(C)$. By Claim $1, d_{G}\left(v_{0}\right)=1$. So by Lemma 2.2.7-(3), $G^{2}$ admits a $3-\mathrm{NZF}$, a contradiction.

Claim 6. Let $e=x y \in E(G)$ with $d_{G}(x)=d_{G}(y)=3$. Then e is contained in a circuit of length 3 or 4 .

By contradiction. Let $G_{1}$ be the graph obtained from $G$ by deleting the edge $e$ and adding a new vertex $y^{\prime}$ and a new edge $x y^{\prime}$. Since $G$ contains no degree 2 vertices and $d_{G_{1}}(y)=2$, then $G_{1} \notin \overline{\mathcal{T}}_{1,3}$. By Claim $1, e$ is not a cut edge of $G$, then by (i), $G_{1}^{2}$ admits a 3 -NZF $\left(D, f_{1}\right)$. Identify $y$ and $y^{\prime}$, the resulting 3 -flow $\left(D, f_{2}\right)$ in $G^{2}$ misses only two edges $y_{1} x$ and $y_{2} x$ where $N(y)=\left\{y_{1}, y_{2}, x\right\}$ (since $x y$ is not contained a circuit of length 3 or 4 ). By Lemma 2.2.5-(2), $G^{2}$ admits a 3-NZF, a contradiction.

Claim 7. For each $x \in V(G)$ with $d_{G}(x)=3,\left|N_{G}(x) \cap V_{3}\right| \leq 2$, where $V_{3}$ is the set of all the degree 3 vertices of $G$.

By contradiction. Assume that $U=\left\{u_{1}, u_{2}, u_{3}\right\}=N_{G}(x) \cap V_{3}$. Let $G_{1}=G \backslash\{x\}$. By Claim $1, G_{1}$ is connected. Since $G$ contains no degree 2 vertices, $G_{1} \notin \overline{\mathcal{T}}_{1,3}$ and $G_{1}^{2}$ admits a 3-NZF $(D, f)$. By Claim 6 , each $x u_{i}(1 \leq i \leq 3)$ is contained a circuit of length at most 4. We consider the following 3 cases. 
Case 1. $G[U]$ contains at least 2 edges.

Suppose that $u_{1} u_{2}, u_{2} u_{3} \in E(G)$. Let $u_{i}^{\prime} \in N_{G}\left(u_{i}\right) \backslash U$ for $i=1$, 3. If $u_{1}^{\prime}=u_{3}^{\prime}$, then $G^{2}\left[U \cup\left\{u_{1}^{\prime}, x\right\}\right] \cong K_{5}$, by Lemma 2.2.5-(1), we can get a 3-NZF of $G^{2}$, a contradiction. If $u_{1}^{\prime} \neq u_{3}^{\prime}$, then $G\left[u_{1}^{\prime} u_{1} u_{2} u_{3} u_{3}^{\prime}\right]$ is a 4-path, by Lemma 2.2.5-(1) (similar to Case 3 of Claim 4), we can get a $3-\mathrm{NZF}$ of $G^{2}$, a contradiction.

Case 2. $G[U]$ contains exactly 1 edge.

Assume that $u_{1} u_{2} \in E(G)$. By Claim 6, each edge $x u_{i}(i=1,2,3)$ is contained in a circuit of length 3 or 4 . So we may assume $z \in\left(N_{G}\left(u_{2}\right) \cap N_{G}\left(u_{3}\right)\right) \backslash\{x\}$. Clearly, $G^{*}=G^{2}[U \cup\{x, z\}] \cong K_{5}$. Let $u_{i}^{\prime} \in N_{G}\left(u_{i}\right) \backslash(U \cup\{z\})$ for $i=1$, 3. Clearly, $E\left(G^{2}\right) \backslash \operatorname{supp}(f) \subseteq$ $E\left(G^{*}\right) \cup\left\{x u_{1}^{\prime}, x u_{3}^{\prime}\right\}$. Since $x u_{j} u_{j}^{\prime} x(j=1,3)$ is a circuit of $G^{2}$, we can get a 3 -flow $\left(D, f_{1}\right)$ such that $E\left(G^{2}\right) \backslash \operatorname{supp}\left(f_{1}\right) \subseteq E\left(G^{*}\right)$. By Lemma 2.2.5-(1), we can get a 3 -NZF of $G^{2}$, a contradiction.

Case 3. $G[U]$ contains no edges.

Assume that $z_{1} \in\left(N_{G}\left(u_{1}\right) \cap N_{G}\left(u_{2}\right)\right) \backslash\{x\}$ and $z_{2} \in\left(N_{G}\left(u_{1}\right) \cap N_{G}\left(u_{3}\right)\right) \backslash\{x\}$. Let $G_{2}=$ $G \backslash\left\{x u_{1}\right\}$, then $G_{2} \notin \overline{\mathcal{T}}_{1,3}$ and $G_{2}^{2}$ admits a $3-\mathrm{NZF}\left(D, f_{1}\right)$. Clearly, $E\left(G^{2}\right) \backslash \operatorname{supp}\left(f_{1}\right)=\left\{x u_{1}\right\}$. Since $x u_{1}$ is contained in a $W_{4}$ which is contained in the graph induced by $\left\{u_{1}, z_{1}, u_{2}, u_{3}, x\right\}$ in $G^{2}$ with $x$ as center, by Lemma 2.2.5-(1), we can get a 3 -NZF of $G^{2}$, a contradiction.

Final Step. By Claim 2, Claim 5 and Claim 7, all vertices of $G$ have degree 1 or 3 and each degree 3 vertex is adjacent to at most 2 degree 3 vertices. So $G\left[V_{3}\right]$ is a path or a circuit, hence $G$ must be a graph obtained from an $r$-circuit $x_{0} x_{1} \cdots x_{r-1} x_{0}$ by attaching an edge $x_{i} v_{i}$ at each $x_{i}$ for $0 \leq i \leq r-1$, where $v_{i} \neq v_{j}$ if $i \neq j$, or a path $x_{0} x_{1} \cdots x_{p}$ by attaching an edge $v_{i} x_{i}(1 \leq i \leq p-1)$ at each $x_{i}$, where $v_{i} \neq v_{j}$ if $i \neq j$. Clearly the latter case is a graph in $\overline{\mathcal{T}}_{1,3}$. By Lemma 2.2.7-(2), $G^{2}$ admits a $3-\mathrm{NZF}$, a contradiction. 


\section{Chapter 3}

\section{Group connectivity of triangularly connected graphs}

Tutte [6] conjectured that every 4 -edge connected graph admits a nowhere-zero $Z_{3}$-flow and Jeager et al [26] conjectured that every 5-edge connected graph is $Z_{3}$-connected. In this paper, we characterize the triangularly connected graphs that are $A$-connected for any Abelian group $A$ with $|A| \geq 3$. As a corollary, we also characterize the triangularly connected graphs that admit nowhere-zero $Z_{3}$-flows, confirming these two conjectures for triangularly connected graphs.

\subsection{Introduction}

For a subset $X \subseteq E(G)$, the contraction $G / X$ is the graph obtained from $G$ by identifying the two ends of each edge in $X$ and then deleting the resulting loops. Note that even when $G$ is simple, $G / X$ may have multiple edges. For convenience, we write $G / e$ for $G /\{e\}$, where $e \in E(G)$. If $H$ is a subgraph of $G$, then $G / H$ denotes $G / E(H)$. 
We use $P_{k}$ for a path of length $k$. A nontrivial 2-regular connected graph is called a circuit and a circuit with $k$ edges is called a $k$-circuit. A wheel $W_{k}$ is a graph obtained from a $k$-circuit by adding a new vertex $v$ and then joining this new vertex to all the vertices on the circuit. This new vertex $v$ is called the center of $W_{k}$. A wheel $W_{k}$ is an even wheel if $k$ is even and an odd wheel otherwise. We use $\left\langle W_{\text {odd }}\right\rangle$ to denote the set of all odd wheels.

A graph $G$ is called a Fan-graph, if it can be obtained from a path $P_{k}=v_{1} v_{2} \cdots v_{k}$ $(k \geq 2)$ by adding a new vertex $v$ and joining $v$ to all the vertices on the path. $v$ is called the center of $G, v v_{1}, v v_{k}$ are called the end edges of $G$ and $v_{1}, v_{k}$ are called the end points of $G$. The set of Fan-graphs are denoted by $\langle F\rangle$.

Let $G$ be a graph with $u^{\prime} v^{\prime} \in E(G)$ and $H$ be a graph with $u v \in E(H)$. We use $G \oplus H$ to denote a new graph obtained from the disjoint union of $G-\left\{u^{\prime} v^{\prime}\right\}$ and $H$ by identifying $u^{\prime}$ and $u$ and identifying $v^{\prime}$ and $v$. This operation is called attaching $G$ on $H$ over the edge $u v$ and the resulting graph is denoted by $G \oplus H$.

We define $\langle W F\rangle=\left\{H \mid H=H_{1} \oplus H_{2} \oplus \cdots \oplus H_{k}\right.$, where $H_{i} \in\langle F\rangle \cup\left\langle W_{\text {odd }}\right\rangle$ for $\left.1 \leq i \leq k\right\}$. We call $H_{i}(1 \leq i \leq k)$ is a WF-component of $H$. An end-WF-component of $H$ is a WF-component $H_{i}$ such that $H_{i}$ shares at most one edge with the union of all the other WF-components of $H$.

Let $G$ be a digraph, $A$ be a nontrivial additive Abelian group and $A^{*}$ be the set of nonzero elements in $A$. We define

$$
F(G, A)=\{f \mid f: E(G) \mapsto A\} \quad \text { and } \quad F^{*}(G, A)=\left\{f \mid f: E(G) \mapsto A^{*}\right\}
$$

For each $f \in F(G, A)$, the boundary of $f$ is a function $\partial f: V(G) \mapsto A$ defined by $\partial f=\sum_{e \in E^{+}(v)} f(e)-\sum_{e \in E^{-}(v)} f(e)$, where " $\sum$ " refers to the addition in $A$. We define $Z(G, A)=\left\{b \mid b: V(G) \mapsto A\right.$ with $\left.\sum_{v \in V(G)} b(v)=0\right\}$.

An undirected graph $G$ is $A$-connected, if $G$ has an orientation $G^{\prime}$ such that for every function $b \in Z(G, A)$, there is a function $f \in F^{*}\left(G^{\prime}, A\right)$ such that $\partial f=b$. For an Abelian 
group $A$, let $\langle A\rangle$ denote the family of graphs that are $A$-connected. It has been observed in [26] that $G \in\langle A\rangle$ is independent of the orientation of $G$.

Clearly, an $A$-nowhere-zero-flow (abbreviated as $A$-NZF) in a digraph $G$ is a function $f \in F^{*}(G, A)$ such that $\partial f=0$.

The concept of $A$-connectivity was introduced by Jaeger et al in [26], which is a generalization to the $A-\mathrm{NZF}$.

Jaeger proposed several conjectures on group connectivity of graphs.

Conjecture 3.1.1 (Jaeger et al [26]) Every 3-edge connected graph is $Z_{5}$-connected.

Conjecture 3.1.2 (Jaeger et al [26]) Every 5-edge connected graph is $Z_{3}$-connected.

A weaker version of the Conjecture 3.1 .2 was also proposed:

Conjecture 3.1.3 (Jaeger et al [26]) There is an integer $k$ such that every $k$-edge connected graph is $Z_{3}$-connected.

Note that Conjecture 3.1.1 is stronger than the 5-flow conjecture, Conjecture 3.1.2 is stronger than the 3-flow conjecture and Conjecture 3.1.3 is equivalent to the weak 3-flow conjecture (see [26]). For these conjectures, Jaeger got some partial results.

Theorem 3.1.4 (Jaeger et al [26]) Every graph which contains 2 edge-disjoint spanning trees (in particular, every 4-edge connected graph) is A-connected for every Abelian group A with $|A| \geq 4$.

Theorem 3.1.5 (Jaeger et al [26]) Every 3-edge connected graph is A-connected for every Abelian group $A$ with $|A| \geq 6$. 
Theorem 3.1.4 and Theorem 3.1.5 are generalizations to Theorem 2.1.7 and Theorem 2.1.5, respectively.

A connected graph $G$ is locally connected if $G[N(v)]$ is connected for every $v \in V(G)$. A graph $G$ is triangularly connected if for every $e_{1}, e_{2} \in E(G)$, there exists a sequence of circuits $C_{1}, C_{2}, \ldots, C_{k}$ such that $e_{1} \in E\left(C_{1}\right), e_{2} \in E\left(C_{k}\right),\left|E\left(C_{i}\right)\right| \leq 3$ for $1 \leq i \leq k$, and such that $E\left(C_{j}\right) \cap E\left(C_{j+1}\right) \neq \emptyset$ for $1 \leq j \leq k-1$. Note that every locally connected graph on at least three vertices is also triangularly connected.

Motivated by the 3-flow conjecture and the conjecture above for $Z_{3}$-connectivity, we study the family of triangularly connected graphs and confirm these two conjectures for this family of graphs.

The following are the main results of this chapter.

Theorem 3.1.6 Let $G$ be a triangularly connected graph. Then $G \in\langle A\rangle$ for all additive Abelian group $A$ with $|A| \geq 3$ if and only if $G \notin\langle W F\rangle$.

As the corollary of Theorem 3.1.6, we get the following result.

Theorem 3.1.7 Let $G$ be a triangularly connected graph. Then $G$ admits a $Z_{3}-N Z F$ if and only if $G \neq G_{1} \oplus G_{2} \oplus \cdots \oplus G_{k}$ such that $G_{i} \in\left\langle W_{\text {odd }}\right\rangle$.

Theorem 3.1.7 is a generalization of Theorem 2.1.16.

Corollary 3.1.8 The 3-flow conjecture is true for the family of triangularly connected graphs.

Proof. By the structure of forbidden graphs in Theorem 3.1.7, every such graph has at least one small degree (less than 4) vertex. Therefore, it is not 4-edge connected. Then by Theorem 3.1.7, the 3-flow conjecture is true for the family of triangularly connected graphs. 


\subsection{Lemmas}

Lemma 3.2.1 (Chen et al [11]) (1) Let $H$ be a subgraph of $G$ and $H \in\left\langle Z_{3}\right\rangle$, then $G / H \in$ $\left\langle Z_{3}\right\rangle$ if and only if $G \in\left\langle Z_{3}\right\rangle$.

(2) If $G \in\left\langle Z_{3}\right\rangle$, then for any $e \in E\left(G^{c}\right), G+e \in\left\langle Z_{3}\right\rangle$, where $G^{c}$ is the complement of $G$.

Lemma 3.2.2 (Lai [32]) (1) Let $C_{n}$ be a $n$-circuit with $n \geq 2$ and let $A$ be an Abelian group. Then, $C_{n} \in\langle A\rangle$ if and only if $|A| \geq n+1$.

(2) Let $T$ be a connected spanning subgraph of $G$. If for each edge $e \in E(T)$, G has a subgraph $H_{e} \in\langle A\rangle$ with $e \in H_{e}$, then $G \in\langle A\rangle$.

Lemma 3.2.3 Let $G \cong W_{n}$ for some integer $n \geq 3$. Then $G$ admit a $Z_{3}-N Z F$ if $n$ is even and $G$ admits no $Z_{3}-N Z F$ if $n$ is odd.

Proof. By Lemma 2.2.4, we need only to show that any even wheel has a modular 3orientation while any odd wheel does not.

Suppose that $G$ is an even wheel with circuit $v_{1}, v_{2}, \cdots v_{2 k} v_{1}$ and center $v_{0}$. Since it is an even wheel, we orient the edges of $G$ such that $v_{i}$ is the head of all the edges incident with it for $i$ is odd. And then orient remaining edges such that $v_{0}$ is the head. It is clear, the resulting orientation is a modular 3-orientation of $G$.

Now suppose that $G$ is an odd wheel with circuit $v_{1}, v_{2}, \cdots v_{2 k+1} v_{1}$ and center $v_{0}$. Since $d_{G}\left(v_{1}\right)=d_{G}\left(v_{2}\right)=\cdots=d_{G}\left(v_{2 k+1}\right)=3$, if there is a modular 3-orientation of $G$, then each vertex $v_{i}(1 \leq i \leq 2 k+1)$ is either the head of all the 3 edges incident with it or the tail of the 3 edges incident with it. But it is impossible because $v_{1}, v_{2}, \cdots v_{2 k+1} v_{1}$ is a circuit with odd number of vertices. Therefore, $G$ admits no $Z_{3}$-NZF. 
Lemma 3.2.4 (1). Let $G \cong W_{2 n}$ for some integer $n \geq 1$. Then $G \in\left\langle Z_{3}\right\rangle$.

(2). Let $G \cong W_{2 n+1}$ be a digraph and $b \in Z\left(G, Z_{3}\right)$. Then there exists an $f \in F^{*}\left(G, Z_{3}\right)$ with $\partial f=b$ if and only if $b \neq \overline{0}$.

Proof. We prove (1) and (2) at the same time by induction on $n$. If $n=1$, then $W_{2 n}$ can be obtained from a 3-circuit by adding a parallel edge. By Lemma 3.2.2-(1) and Lemma 3.2.1(1), it is $Z_{3}$-connected.

For the sake of convenience, let $v_{1}, v_{2} \cdots, v_{m} v_{1}$ be the circuit of $W_{m}$ and $v_{0}$ be its center. Edges are oriented so that $v_{1} v_{2} \cdots v_{m} v_{1}$ is a directed circuit and all the other edges with $v_{0}$ as the head.

By induction hypothesis, we may assume that Lemma 3.2.4 holds for $W_{k}$ with $k \leq 2 n$.

(I). Let us consider $G \cong W_{2 n+1}$ first.

$\Longrightarrow$ By contradiction. Suppose that $b=\overline{0}$. There exists an $f \in F^{*}\left(G, Z_{3}\right)$ with $\partial f=$ $b=\overline{0}$. This means $G \cong W_{2 n+1}$ admits a $Z_{3}-\mathrm{NZF}$, which is impossible by Lemma 3.2.3. So $b \neq \overline{0}$.

$\Longleftarrow$ Suppose that $b \neq \overline{0}$. Without loss of generality, we may assume $b\left(v_{2 n+1}\right) \neq 0$. Then let $G^{\prime}=G \backslash\left\{v_{0} v_{2 n+1}\right\}$ and define

$$
b^{\prime}(v)= \begin{cases}b(v) & \text { if } v \notin\left\{v_{0}, v_{2 n+1}\right\}, \\ b(v)+b\left(v_{2 n+1}\right) & \text { if } v=v_{0}, \\ 0 & \text { if } v=v_{2 n+1} .\end{cases}
$$

Let $G^{\prime \prime}=G^{\prime} \backslash\left\{v_{1} v_{2 n+1}, v_{2 n+1} v_{2 n}\right\} \cup\left\{v_{1} v_{2 n}\right\}$ and orient the edge $v_{1} v_{2 n}$ as $v_{2 n} \rightarrow v_{1}$. Let $b^{\prime \prime}$ be the restriction of $b^{\prime}$ to $V\left(G^{\prime \prime}\right)$. Clearly, $b^{\prime \prime} \in Z\left(G^{\prime \prime}, Z_{3}\right)$ and $G^{\prime \prime} \cong W_{2 n}$. By induction hypothesis, there exists an $f^{\prime \prime} \in F^{*}\left(G^{\prime \prime}, Z_{3}\right)$ with $\partial f^{\prime \prime}=b^{\prime \prime}$. Now let us define

$$
f^{\prime}(e)= \begin{cases}f^{\prime \prime}(e) & \text { if } e \neq v_{1} v_{2 n} \\ f^{\prime \prime}\left(v_{1} v_{2 n}\right) & \text { if } e \in\left\{v_{2 n} v_{2 n+1}, v_{2 n+1} v_{1}\right\}\end{cases}
$$


By the relation between $G^{\prime \prime}$ and $G^{\prime}$, we have $f^{\prime} \in F^{*}\left(G^{\prime}, Z_{3}\right)$ and $\partial f^{\prime}=b^{\prime}$. Clearly this $f^{\prime}$ can be extended to an $f \in F^{*}\left(G, Z_{3}\right)$ with $\partial f=b$ by defining

$$
f(e)= \begin{cases}f^{\prime}(e) & \text { if } e \neq v_{0} v_{2 n+1}, \\ b\left(v_{2 n+1}\right) & \text { if } e=v_{0} v_{2 n+1} .\end{cases}
$$

(II). Now let us consider $G \cong W_{2 n+2}$. Let $b \in Z\left(G, Z_{3}\right)$.

Case 1. $b=\overline{0}$.

By Lemma 3.2.3, $W_{2 n+2}$ admits a $Z_{3}$-NZF, then there exists an $f \in F^{*}\left(G, Z_{3}\right)$ with $\partial f=\overline{0}$.

Case 2. Exactly one of $b\left(v_{i}\right)(1 \leq i \leq 2 n+2)$ is not 0 .

Suppose that $b\left(v_{1}\right)=i \neq 0$, then $b\left(v_{0}\right)=-i$ and $b\left(v_{j}\right)=0$ for $2 \leq j \leq 2 n+2$. Let us first reorient the edges to get a digraph $G^{\prime}$ as follows: Orient $v_{0} v_{1}$ as $v_{0} \rightarrow v_{1}$ and let $v_{k}$ be the tail of all the other edges incident with it if $k$ is odd, let $v_{k}$ be the head of all the edges incident with it if $k$ is even and not zero. Define $f^{\prime}: E\left(G^{\prime}\right) \mapsto Z_{3}$ as $f(e)=i$ for each $e \in E\left(G^{\prime}\right)$. It is easy to verify that $f^{\prime} \in F^{*}\left(G^{\prime}, Z_{3}\right)$ with $\partial f^{\prime}=b$. Now, we can get an $f \in F^{*}\left(G, Z_{3}\right)$ with $\partial f=b$ by defining

$$
f(e)= \begin{cases}f^{\prime}(e) & \text { if } e \text { has the same orientation in } G \text { as in } G^{\prime}, \\ -f^{\prime}(e) & \text { otherwise. }\end{cases}
$$

Case 3. At least two of $b\left(v_{i}\right)(1 \leq i \leq 2 n+2)$ are not 0 .

Without loss of generality, we may assume $b\left(v_{2 n+2}\right) \neq 0$. Then let $G^{\prime}=G \backslash\left\{v_{0} v_{2 n+2}\right\}$ and define

$$
b^{\prime}(v)= \begin{cases}b(v) & \text { if } v \notin\left\{v_{0}, v_{2 n+2}\right\}, \\ b(v)+b\left(v_{2 n+2}\right) & \text { if } v=v_{0}, \\ 0 & \text { if } v=v_{2 n+2} .\end{cases}
$$

Let $G^{\prime \prime}=G^{\prime} \backslash\left\{v_{1} v_{2 n+2}, v_{2 n+2} v_{2 n+1}\right\} \cup\left\{v_{1} v_{2 n+1}\right\}$ and orient the edge $v_{1} v_{2 n+1}$ as $v_{2 n+1} \rightarrow v_{1}$. Let $b^{\prime \prime}$ be the restriction of $b^{\prime}$ to $V\left(G^{\prime \prime}\right)$. Then $b^{\prime \prime} \in Z\left(G^{\prime \prime}, Z_{3}\right), G^{\prime \prime} \cong W_{2 n+1}$ and $b^{\prime \prime} \neq \overline{0}$. By 
(I) there exists an $f^{\prime \prime} \in F^{*}\left(G^{\prime \prime}, Z_{3}\right)$ with $\partial f^{\prime \prime}=b^{\prime \prime}$. Similar to the proof in (I), we can get an $f \in F^{*}\left(G, Z_{3}\right)$ with $\partial f=b$.

Lemma 3.2.5 Let $G$ be a triangularly connected graph. If $H$ is a non-trivial subgraph of $G$ and $H \in\left\langle Z_{3}\right\rangle$, then $G \in\left\langle Z_{3}\right\rangle$.

Proof. Let $H^{\prime}$ be a subgraph of $G$ with $E(H) \subseteq E\left(H^{\prime}\right)$ such that $H^{\prime} \in\left\langle Z_{3}\right\rangle$ and $\left|E\left(H^{\prime}\right)\right|$ as large as possible. We are to show that $H^{\prime}=G$. Assume not, let $e \in E(G) \backslash E\left(H^{\prime}\right)$ and $e^{\prime} \in E\left(H^{\prime}\right)$. Let $C_{1}, C_{2}, \cdots C_{k}$ be a triangle chain joining $e^{\prime}$ and $e$ in $G$ with $e^{\prime} \in C_{1}$ and $e \in C_{k}$. Let $i$ be the smallest subscribe such that $C_{i} \nsubseteq E E\left(H^{\prime}\right)$. Note that $C_{i}$ shares at least one edge with $H^{\prime}$. Therefore, if we let $H^{\prime \prime}=H^{\prime} \cup C_{i}$, then $H^{\prime \prime} / H^{\prime}$ is a 2-circuit a loop which is $Z_{3}$-connected. Then by Lemma 3.2.1-(1), $H^{\prime \prime} \in\left\langle Z_{3}\right\rangle$, contrary to the choice of $H^{\prime}$.

Lemma 3.2.6 Let $G$ be a graph. Then $G \in\left\langle Z_{3}\right\rangle$ if and only if $G \oplus W_{2 n+1} \in\left\langle Z_{3}\right\rangle$ for any integer $n \geq 1$.

Proof. If $G \in\left\langle Z_{3}\right\rangle$, then $G \oplus W_{2 n+1} / G$ is a triangularly connected graph containing a 2circuit. By Lemma 3.2.5, $G \oplus W_{2 n+1} / G \in\left\langle Z_{3}\right\rangle$. Therefore by Lemma 3.2.1-(1), $G \oplus W_{2 n+1} \in$ $\left\langle Z_{3}\right\rangle$.

Conversely, for any $b \in Z\left(G, Z_{3}\right)$, let us define $b^{*} \in Z\left(G \oplus W_{2 n+1}, Z_{3}\right)$ as follows:

$$
b^{*}(v)= \begin{cases}b(v) & \text { if } v \in V(G), \\ 0 & \text { otherwise }\end{cases}
$$

Since $G \oplus W_{2 n+1} \in\left\langle Z_{3}\right\rangle$, then there exists $f^{*} \in F^{*}\left(G \oplus W_{2 n+1}, Z_{3}\right)$ such that $\partial f^{*}=b^{*}$. Let $f$ be the restriction of $f^{*}$ to $E(G)$. We will show that $f \in F^{*}\left(G, Z_{3}\right)$ and $\partial f=b$.

Let $v_{0}$ be the center of $W_{2 n+1}$ and $v_{1} v_{2} \in E(G) \cap E\left(W_{2 n+1}\right)$. Without loss of generality, let $v_{0} v_{2}, v_{1} v_{2}, v_{3} v_{2}$ be oriented so that $v_{2}$ is the head of these three edges. 
If $f^{*}\left(v_{0} v_{2}\right)+f^{*}\left(v_{1} v_{2}\right)+f^{*}\left(v_{3} v_{2}\right)=\alpha \in Z_{3} \backslash\{0\}$ then change $f\left(v_{1} v_{2}\right)$ to be $\alpha$. It is easy to see that $f \in F^{*}\left(G, Z_{3}\right)$ and $\partial f=b$. So assume that $\alpha=0 \in Z_{3}$. In this case, it is also easy to see that the restriction of $f^{*}$ on $W_{2 n+1}$ is a 3-NZF which contradicts Lemma 3.2.4-(2). Therefore $G \in\left\langle Z_{3}\right\rangle$.

Lemma 3.2.7 (Devos et al [12]) Let $G_{1}, G_{2}$ be graphs and let $H=G_{1} \oplus G_{2}$. If neither $G_{1}$ or $G_{2}$ is $Z_{3}$-connected, then $H$ is not $Z_{3}$-connected.

Lemma 3.2.8 Let $H \in\langle F\rangle$. Then $H \notin\left\langle Z_{3}\right\rangle$.

Proof. Since $H \in\langle F\rangle, H$ is a nontrivial subgraph of $W_{2 n+1}$ for some integer $n$. By Lemma 3.2.4-(2), $W_{2 n+1} \notin\left\langle Z_{3}\right\rangle$. Then by Lemma 3.2.5, $H \notin\left\langle Z_{3}\right\rangle$.

Lemma 3.2.9 Let $G=G_{1} \cup G_{2}$ be a graph so that $G_{1}$ and $G_{2}$ are two distinct wheels. If $\left|E\left(G_{1}\right) \cap E\left(G_{2}\right)\right| \geq 2$, then $G \in\left\langle Z_{3}\right\rangle$.

Proof. Clearly, $G$ is a triangularly connected. If one of $G_{i}$ is an even wheel, then by Lemma 3.2.4-(1) and Lemma 3.2.5, $G \in\left\langle Z_{3}\right\rangle$.

So we may assume both $G_{1}$ and $G_{2}$ are odd wheels. Since $\left|E\left(G_{1}\right) \cap E\left(G_{2}\right)\right| \geq 2$, let $e_{1}=u_{1} u_{2}, e_{2}=w_{1} w_{2}$ be two edges in $E\left(G_{1}\right) \cap E\left(G_{2}\right)$. If the edge induced graph $G\left[\left\{e_{1}, e_{2}\right\}\right]$ has a degree one vertex which is not the center of neither $G_{1}$ nor $G_{2}$, then let $v$ be that vertex; Otherwise, $e_{1}, e_{2}$ must share one vertex, say $u_{2}=w_{2}$, and $u_{1}, w_{1}$ are centers of $G_{1}$ and $G_{2}$ respectively. Then $u_{1} w_{1} \in E\left(G_{1}\right) \cap E\left(G_{2}\right)$. For this case, let $v=u_{2}$. Thus for all these two cases, $G_{1} \backslash v$ and $G_{2} \backslash v$ still share at least one edge. Clearly, both $G_{1} \backslash v$ and $G_{2} \backslash v$ are triangularly connected. Therefore $G \backslash v$ is triangularly connected.

Clearly, $d_{G}(v) \geq 4$. Let $N_{G}(v)=\left\{v_{1}, v_{2}, \cdots, v_{k}\right\}$ with $k \geq 4$. Since $G$ is triangularly connected, $v$ must be contained in some triangle, say $v v_{1} v_{2} v$. Let $G^{*}$ be a new graph obtained 
from $G$ by deleting $v$ and adding a parallel edge $v_{1} v_{2}$. Because $G \backslash v$ is triangularly connected, $G^{*}$ remains triangularly connected and contains a 2-circuit. By Lemma 3.2.5, $G^{*} \in\left\langle Z_{3}\right\rangle$. By Lemma 3.2.2-(1) and Lemma 3.2.1-(1), $G^{*} \cup\left\{v v_{3}, v v_{4}, \cdots, v v_{k}\right\} \in\left\langle Z_{3}\right\rangle$. Then it follows $G \in\left\langle Z_{3}\right\rangle$.

\subsection{Proof of the theorem}

Proof of Theorem 3.1.6. Note that since $G$ is a triangularly connected graph, each edge of $G$ is contained in at least one circuit $C$ with $|E(C)| \leq 3$, then by Lemma 3.2.2-(1) and (2), $G \in\langle A\rangle$ for any Abelian group $A$ with $|A| \geq 4$. So we need only to prove the theorem for $A=Z_{3}$.

$\Longrightarrow$ By contradiction. Suppose that $G \in\langle W F\rangle$. By Lemma 3.2.8, for any $H \in\langle F\rangle$, we have $H \notin\left\langle Z_{3}\right\rangle$ and by Lemma 3.2.4-(2), we have $W_{2 n+1} \notin\left\langle Z_{3}\right\rangle$. Therefore by Lemma 3.2.7, $G \notin\left\langle Z_{3}\right\rangle$, a contradiction.

$\Longleftarrow$ By contradiction. Let $G$ be a graph such that

(1) $G$ is a counterexample to Theorem 3.1.6, i.e. $G$ is triangularly connected, $G \notin\langle W F\rangle$ and $G \notin\left\langle Z_{3}\right\rangle$.

(2) Subjected to (1), $|E(G)|$ is minimized.

Claim 1. The girth of $G$ is 3 and $\delta(G) \geq 3$.

Clearly, $G \nsucceq K_{3}$. It is easy to see that $G$ contains no 2-circuits. Otherwise, if $G$ contains a 2-circuit $C$, then by Lemma 3.2.2-(1) and Lemma 3.2.5, $G \in\left\langle Z_{3}\right\rangle$, contrary to (1).

If there exists $v \in V(G)$ such that $d_{G}(v)=2$, then let $C=v x y v$ be a triangle containing the vertex $v$. Since $G$ contains no 2-circuits, $C$ is the only triangle containing $v x$ (and $v y$ as well). Therefore $G \backslash v$ remains triangularly connected. 
If $G \backslash v \in\left\langle Z_{3}\right\rangle$, then it follows $G \in\left\langle Z_{3}\right\rangle$, contrary to (1); If $G \backslash v \notin\left\langle Z_{3}\right\rangle$, then either $G \backslash v \in\langle W F\rangle$ or it is contrary to (2). Suppose that $G \backslash v \in\langle W F\rangle$. Then $G=(G \backslash v) \oplus C$, that is, $G \in\langle W F\rangle$, contrary to $(1)$. So $\delta(G) \geq 3$.

Claim 2. Let $H$ be a maximal triangularly connected subgraph of $G$ such that $|E(H)|<$ $|E(G)|$. Then $|E(G)|-|E(H)|=1$ and $V(G)=V(H)$.

A proper subgraph of $G$ that is triangularly connected exists since any triangle of $G$ is a subgraph of this kind.

If $|E(G)|-|E(H)|$ has more than two edges, then there is an edge $e \in H$ that is contained in a triangle $C$ such that $E(C) \backslash E(H) \neq \emptyset$ since $G$ is triangularly connected.

So, adding $E(C) \backslash E(H) \neq \emptyset$, which consists of at most two edges, into $H$. Repeat this procedure, we are able to obtain the maximal one $H$. Clearly, $|E(G)|-|E(H)| \leq 2$.

If $|E(G)|-|E(H)|=2$, the two edges in $E(G) \backslash E(H)$ must share one vertex, we may assume these two edges are $v x, v y$. Clearly, $x y \in E(H)$. If $v \in V(G) \backslash V(H)$, then $d_{G}(v)=2$, contrary to Claim 1 . So $V(H)=V(G)$. Clearly, $d_{G}(v) \geq 4$. Delete $v x, v y$ from $G$ and add a parallel edge to $x y$, the resulting graph, call it $H^{*}$, is triangularly connected and contains a 2-circuit. Then by Lemma 3.2.2-(1) and Lemma 3.2.5, $H^{*} \in\left\langle Z_{3}\right\rangle$. Therefore $G \in\left\langle Z_{3}\right\rangle$, contrary to $(1)$.

Then $|E(G)|-|E(H)|=1$. By Claim $1, \delta(G) \geq 3$, then clearly $V(G)=V(H)$.

The last one edge of $E(G) \backslash E(H)$ will add the last triangle (say, $C=x y z x$ ) into $H$. For convenience, we may assume that $e=x z \in E(G) \backslash E(H)$.

Claim 3. $H \in\langle W F\rangle$.

Otherwise, by the choice of $G, H \in\left\langle Z_{3}\right\rangle$. Then $G / H$ is a loop which is $Z_{3}$-connected. By Lemma 3.2.1-(1), $G \in\left\langle Z_{3}\right\rangle$, contrary to the choice of $G$. Therefore $H \in\langle W F\rangle$.

Claim 4. $H$ contains exactly two end-WF-components and one end-WF-component con- 
tains $y x$, the other one contains $y z$.

If $H$ contains exactly one WF-component, then $H$ is either a Fan-graph or an odd wheel. Suppose that $H$ is a Fan-graph. Since $\delta(G) \geq 3$, then $x$ and $z$ will be the end points of $H$, therefore $G$ will be a wheel, contrary to (1). If $G$ is an odd wheel, then $G=H \cup e$ contains an even wheel. By Lemma 3.2.4-(1) and Lemma 3.2.5, $G \in\left\langle Z_{3}\right\rangle$, contrary to (1).

Then we will show that $H$ contains exactly two end-WF-components. Otherwise, since $E(G) \backslash E(H)=\{x z\}, y x, y z \in E(H)$ and $\delta(G) \geq 3$, then there exists an end-WF-component $H^{\prime} \in\left\langle W_{\text {odd }}\right\rangle$ such that $H=H^{*} \oplus H^{\prime}$ with $H^{*} \in\langle W F\rangle$ and $\{x, y, z\} \cap\left(V\left(H^{\prime}\right) \backslash V\left(H^{*}\right)\right)=\emptyset$. Therefore $G=\left(H^{*} \cup e\right) \oplus H^{\prime}$. Since $G \notin\langle W F\rangle, H^{*} \cup e \notin\langle W F\rangle$. Since $\{x, y, z\} \subseteq V\left(H^{*}\right)$ and $H^{*} \in\langle W F\rangle$, then $H^{*} \cup e$ is triangularly connected. By Lemma 3.2.6, $H^{*} \cup e$ is a smaller counterexample, contrary to $(2)$.

The WF-component of $H$ containing $y x$ must be an end-WF-component. Otherwise, since there are two end-WF-components of $H$, then at least one of them, say $H_{1}$, has the similar property as $H^{\prime}$ mentioned above. By the same argument, we can get a contradiction. Similarly, the WF-component of $H$ containing $y z$ is the other end-WF-component.

Final Step. By Claim 4, let $H_{1}, H_{2}$ be the two end-WF-components of $H$. We may assume that $y x \in E\left(H_{1}\right), y z \in E\left(H_{2}\right)$. Clearly, $H_{1}$ will not share $y x$ with other WFcomponents of $H$. Otherwise, $H_{1}$ would have the similar property as $H^{\prime}$ in Claim 4 . Similarly, $H_{2}$ will not share $y z$ with other WF-components of $H$.

Since $H_{1}, H_{2}$ are the only two end-WF-components of $H$, both containing vertex $y$. Then every other WF-component of $H$ must contain $y$, too. Clearly, no edges are contained in more than two WF-components of $H$ (otherwise, $H$ would have more than two end-WFcomponents) and if an edge is contained in two WF-components of $H$, then it must have $y$ as one end.

Since $\delta(G) \geq 3$, then each WF-component which belongs to $\langle F\rangle$ must have $y$ as its center. 
By the structure of $H$, there is a path in $N_{H}(y)$ connecting $x$ and $z$, say, $P=x u_{1} u_{2} \cdots u_{k} z$, such that $P$ contains at least one edge of each WF-component of $H$. Then $H[V(P) \cup\{y\}]$ contains a subgraph $H_{4} \in\langle F\rangle$ with $y$ as its center and $y x, y z$ as its end edges. Therefore, $H_{5}=H_{4} \cup\{x z\}$ is a wheel. Clearly $H_{5}$ contains at least two edges of each WF-component of $H$ because $P$ contains at least one edge of each WF-component.

We claim that $H$ contains at least one odd wheel, say $H_{6}$, as its WF-component. Otherwise, each WF-component belongs to $\langle F\rangle$ and $y$ is the center of each WF-component. Then $H \in\langle F\rangle$ with $y$ as it center and $H$ has only one WF-component, a contradiction.

Clearly $H_{5}$ and $H_{6}$ are two distinct wheels sharing at least two edges, by Lemma 3.2.9, $H_{5} \cup H_{6} \in\left\langle Z_{3}\right\rangle$. By Lemma 3.2.5, $G \in\left\langle Z_{3}\right\rangle$, contrary to (1). And this ends the proof of the Theorem 3.1.6.

Proof of Theorem 3.1.7. Similarly to the proof of Lemma 3.2.6, we can get the following result:

A graph $H$ admits a $Z_{3}$-NZF if and only if $H \oplus W_{2 k+1}$ admits a $Z_{3}$-NZF for any integer $k \geq 1$.

Also it is easy to see that for any $H \in\langle F\rangle, H$ admits a $Z_{3}$-NZF, therefore this theorem is a corollary of Theorem 3.1.6. 


\section{Chapter 4}

\section{On circular flows of graphs}

In this chapter, we obtain some sufficient conditions for graphs with circular flow index less than 4. As an immediate corollary, we give a simple proof of a result obtained recently by Galluccio and Goddyn (Combinatorica, 2002), and obtain a larger family of such graphs.

\subsection{Introduction}

The concept of circular flow, a dual version of circular coloring, was introduced by Goddyn et. al. [16]. (Note that the circular flow problem is not a fractional version of the integer flow problem since it is a real line extension instead of a linear programming relaxation).

Definition 4.1.1 Let $G=(V, E)$ be a finite 2-edge connected graph. The circular flow index $\phi_{c}(G)$ of $G$ is defined by

$$
\phi_{c}(G)=\min _{D} \max _{\emptyset \neq X \subset V} \frac{|\delta X|}{\left|\delta^{+} X\right|}
$$

where $D$ ranges over the strong orientations of $G, \delta^{+} X$ denotes the set of arcs from $X$ to $V-X$ and $\delta X=\delta^{+} X \cup \delta^{+}(V-X)$. 
The smallest integer $k$ such that $G$ admits a $k$-NZF is called the flow index of $G$, denoted by $\phi(G)$. Note that $\phi(G)=\left\lceil\phi_{c}(G)\right\rceil$ (see [16]). Therefore, circular flow index can be considered as a refinement of flow index and all the flow conjectures can be restated as follows:

The 5-flow Conjecture (Tutte [46]) Let $G$ be a 2-edge connected graph, then $\phi_{c}(G) \leq 5$.

The 4-flow Conjecture (Tutte [48]) Let $G$ be a 2-edge connected graph containing no subdivision of the Petersen graph, then $\phi_{c}(G) \leq 4$.

The 3-flow Conjecture (Tutte, unsolved problem 48 in [6]) Let $G$ be a 4-edge-connected graph, then $\phi_{c}(G) \leq 3$.

Recently, Galluccio and Goddyn ([15]) proved the following result which is a new approach to the 3 -flow conjecture in the direction of circular flows.

Theorem 4.1.2 (Galluccio and Goddyn [15]) Let $G$ be a 6-edge connected graph. Then $\phi_{C}(G)<4$.

Linear programming methods were applied in the original proof of Theorem $4.1 .2([15])$. In this chapter, we will prove some sufficient conditions for graphs with $\phi_{C}(G)<4$. These results (Theorem 4.3.1 and 4.3.2) are to be proved in purely combinatorial/structural methods and Theorem 4.1.2 will follow immediately. The proof of Theorem 4.1.2 that we will provide here is simpler than the original one in [15].

Now let us introduce some notations and terminology for this chapter. Let $G$ be a graph and $H$ be a subgraph of $G$. For a vertex $v \in V(G)$, the number of edges of $H$ incident with $v$ is denoted by $d_{H}(v)$.

A cycle $C$ is a subgraph $G$ such that $d_{C}(v) \equiv 0(\bmod 2)$. A parity subgraph $P$ of $G$ is a subgraph of $G$ such that $d_{P}(v) \equiv d_{G}(v)(\bmod 2)$ for every vertex $v$ of $G$. A decomposition of a graph $G$ is called a parity subgraph decomposition provided each member of the decomposition 
is a parity subgraph of $G$. A parity subgraph decomposition of $G$ is called trivial if it has only one member (the graph $G$ itself).

Let $T \subseteq V(G)$. A $T$-join in $G$ is a subset $J \subseteq E(G)$ such that $T$ is the set of all odd-degree vertices in the induced subgraph $G[J]$.

A graph $H$ is collapsible if and only if, for every $T$ with $|T| \equiv 0(\bmod 2), H$ contains a connected and spanning $T$-join $J$ in $H$.

We refer readers to [6] or [53] for terminology and notation not defined in this chapter.

\subsection{Lemmas}

Lemma 4.2.1 (Hoffman [19]) Let $G$ be a 2-edge connected graph, $D$ be an orientation of $G$ and $a, b$ be two positive integers $(a \leq b)$. The following two statements are equivalent.

$$
\frac{a}{b} \leq \frac{\left|\delta^{+} X\right|}{\left|\delta^{+}(V(G)-X)\right|} \leq \frac{b}{a}
$$

for each $\emptyset \subset X \subset V(G)$;

(2) $G$ admits a nowhere-zero integer flow $(D, f)$ such that $a \leq f(e) \leq b$ for each $e \in$ $E(G)$.

Lemma 4.2.2 (Nash-Williams [35], Tutte [47], or see [18], [30], [39]) Every 2k-edge connected graph contains $k$ edge-disjoint spanning trees.

Lemma 4.2.3 (Itai and Rodeh [21]) Every spanning tree contains a parity subgraph. 


\subsection{Main results}

The following are the main results of this chapter. With these results, we will give a combinatorial, simple proof of Theorem 4.1.2 and find a large family of graphs with circular flow index less than 4 .

Theorem 4.3.1 Let $G$ be a graph and $(D, f)$ be a nowhere-zero 4-flow of $G$. Let $E_{f=i}=$ $\{e \in E(G): f(e)=i\}$ for $i= \pm 1, \pm 2, \pm 3$. If $\delta(X) \cap E_{f= \pm 2} \neq \emptyset$ for every $X: \emptyset \subset X \subset V(G)$ with $|\delta(X)| \equiv 0(\bmod 4)$, then $\phi_{c}(G)<4$.

Proof. It is sufficient to consider only positive flow since a positive flow can be obtained from a nowhere-zero flow by reversing orientations of some edges and changing the corresponding function value signs. For a positive 4 -flow $(D, f)$ of $G$, it is easy to verify that $D$ is a strong orientation, that is, each edge is contained in a directed circuit. For each nonempty $X \subset V(G)$, since $(D, f)$ is a positive 4-flow, by Lemma 4.2.1, we have $\delta(X) / \delta^{+}(X) \leq 4$. Suppose there exists $X \subseteq V(G)$ such that $\delta(X) / \delta^{+}(X)=4$. Consider the distribution ratio of $\delta^{+}(X) \cap E_{f=i}$ and $\delta^{+}(V-X) \cap E_{f=i}$, for each $i \in\{1,2,3\}$ by applying the equation

$$
\sum_{e \in \delta^{+}(X)} f(e)=\sum_{e \in \delta^{+}(V-X)} f(e)
$$

then we must have that $\delta^{+}(X) \subseteq E_{f=3}, \delta^{+}(V-X) \subseteq E_{f=1}$ and $|\delta(X)| \equiv 0(\bmod 4)$. This contradicts with the fact $\delta(X) \cap E_{f= \pm 2} \neq \emptyset$ (for $\left.i=1,2\right)$. Hence, for each $X \subseteq V(G)$, $\delta(X) / \delta^{+}(X)<4$ which implies $\phi_{c}(G)<4$.

Clearly, similar result can be obtained for $\phi_{c}(G)<k$ for any integer $k>3$.

Theorem 4.3.2 Let $G$ be a graph. If $G$ has a nontrivial parity subgraph decomposition such that at least one of its members is connected and spanning, then $\phi_{c}(G)<4$.

Proof. Let $\left\{P_{1}, P_{2}, P_{3}, \ldots\right\}$ be a non-trivial parity subgraph decomposition of $G$ with $P_{3}$ connected and spanning. Let $C_{1}=P_{1} \cup P_{2}, C_{2}=G \backslash E\left(P_{1}\right)$. Clearly, both $C_{1}$ and $C_{2}$ are 
cycles with $E\left(C_{1}\right) \cup E\left(C_{2}\right)=E(G)$. Let $\left(D, f_{i}\right)$ be a 2-flow of $G$ with $\operatorname{supp}\left(f_{i}\right)=E\left(C_{i}\right)$. Then $(D, f)=\left(D, f_{1}+2 f_{2}\right)$ is a nowhere-zero 4-flow of $G$. For each subset $X: \emptyset \subset X \subset V(G)$, $\delta(X) \backslash E\left(C_{1}\right) \neq \emptyset$ since the spanning connected subgraph $P_{3}$ is contained in $C_{2}-C_{1}$. Hence, $\delta(X) \cap E_{f= \pm 2} \neq \emptyset$. By Theorem 4.3.1, we have that $\phi_{c}(G)<4$.

Proof of Theorem 4.1.2. Since $G$ is 6-edge connected, by Lemma 4.2.2, let $T_{1}, T_{2}, T_{3}$ be three edge disjoint spanning trees of $G$. By Lemma 4.2.3, we can let $P_{i}$ be a parity subgraph of $T_{i}$ (for $i=1,2$ only). Then we have a parity subgraph decomposition $\left\{P_{1}, P_{2}, G \backslash\left(P_{1} \cup P_{2}\right)\right\}$ described in Theorem 4.3.2, where $G \backslash\left(P_{1} \cup P_{2}\right) \supseteq T_{3}$ is connected and spanning.

The concept of collapsible graphs and its corresponding reduction methods were introduced by Catlin ([8]). Note that collapsible graphs contain spanning, connected $T$-joins (including parity subgraphs). Many families of collapsible graphs have been discovered. For example (see [8], [10]),

(1). Graphs containing two edge-disjoint spanning trees,

(2). 2-edge connected graphs containing two edge-disjoint spanning forests $F_{1}$ and $F_{2}$ such that $F_{1}$ is a tree and $\left|E\left(F_{2}\right)\right| \geq|V(G)|-2$,

(3). 2-edge connected graphs containing two edge-disjoint spanning forests $F_{1}$ and $F_{2}$ such that $\left|E\left(F_{1}\right)\right|+\left|E\left(F_{2}\right)\right| \geq 2(|V(G)|-2)$ and $G$ is not contractible to $K_{2, t}$ for some $t \geq 3$,

(4). Graphs in which every edge is contained in a triangle.

The family of graphs described in the following corollary is bigger than that in Theorem 4.1.2.

Corollary 4.3.3 If a graph contains two edge-disjoint subgraphs $P$ and $H$ such that $P$ is a parity subgraph and $H$ is a connected, spanning collapsible subgraph of $G$, then $\phi_{C}(G)<4$.

Proof. Let $H^{\prime}=G-E(P)$. Here $H^{\prime}(\supseteq H)$ is a cycle and remains connected, spanning and collapsible in $G$. Let $P^{\prime}$ be a connected, spanning parity subgraph of $G$ contained in $H^{\prime}$. 
Then we obtain a parity subgraph decomposition $\left\{H^{\prime}-P^{\prime}, P, P^{\prime}\right\}$ described in Theorem 4.3.2. 


\section{Chapter 5}

\section{On flows of bidirected graphs}

Bouchet [7] conjectured that every bidirected graph which admits a nowhere-zero bidirected flow also admits a nowhere-zero bidirected 6 -flow. He proved that it is true with 6 replaced by 216. Zyka [56] proved in his Ph.D dissertation that this conjecture is true with 6 replaced by 30 . Khelladi [28] proved it is true with 6 replaced by 18 for 4-connected graph. In this chapter, we prove that Bouchet's conjecture is true for 6-edge connected bidirected graphs.

\subsection{Introduction}

A bidirected graph $G$ is a graph with vertex set $V(G)$ and edge set $E(G)$ such that each edge is oriented as one of the four possibilities: $\bullet, \bullet, \bullet, \bullet, \ldots, \bullet, \ldots$. An edge with orientation $\bullet . .($ resp. $\bullet .$.$) is called an in-edge (resp. out-edge).$ Edges with other orientations are called ordinary edges. The set of in(out)-edges is denoted by $E_{i}(G)\left(E_{o}(G)\right.$, respectively). We define $O(G)=\left|E_{i}(G)\right|+\left|E_{o}(G)\right|$.

For an ordinary edge $e$, reversing the orientation of $e$ means the natural way to change its orientation. For any non-ordinary edge $e \in E(G)$, reversing the orientation of $e$ means 
changing $e$ from an in(out)-edge to an out(in)-edge. Note that after reversing the orientation of an edge of $G, O(G)$ remains the same.

Let $G$ be a bidirected graph, for any $v \in V(G)$, the set of all edges with tails (or heads) at $v$ is denoted by $E^{+}(v)$ (or $E^{-}(v)$ ) and we define $E(v)=E^{+}(v) \cup E^{-}(v)$ (For general graph $G$, we use $E(v)$ to denote the set of edges which are incident with $v$ in $G$ ). Readers are referred to [6] for terminology not defined in this chapter.

Definition 5.1.1 Let $G$ be a bidirected graph and $f$ be a function: $E(G) \mapsto Z$. Then

(1) $f$ is called a bidirected $k$-flow of $G$ if $-k+1 \leq f(e) \leq k-1$ for every edge $e \in E(G)$ and $\sum_{e \in E^{+}(v)} f(e)=\sum_{e \in E^{-}(v)} f(e)$ for every $v \in V(G)$;

(2) $f$ is called a bidirected modular $k$-flow of $G$ if $0 \leq f(e) \leq k-1$ for every edge $e \in E(G)$ and $\sum_{e \in E^{+}(v)} f(e) \equiv \sum_{e \in E^{-}(v)} f(e)(\bmod k)$ for every $v \in V(G)$;

(3) The support of a bidirected $k$-flow (resp. modular $k$-flow) $f$ of $G$ is the set of edges of $G$ with $f(e) \neq 0($ resp. $f(e) \not \equiv 0(\bmod k))$, and is denoted by $\operatorname{supp}(f)$. A bidirected $k$-flow (modular $k$-flow) of $G$ is nowhere-zero if $\operatorname{supp}(f)=E(G)$.

For nowhere-zero bidirected integer flows, Bouchet [7] proposed the following conjecture (see also Toft and Jensen's book [44]).

Conjecture 5.1.2 Every bidirected graph which admits a nowhere-zero bidirected flow also admits a nowhere-zero bidirected 6-flow.

Note that the value 6 in Conjecture 5.1.2 is best possible: see [7] for details. The following is a list of some partial results for Conjecture 5.1.2.

Theorem 5.1.3 Let $G$ be a bidirected graph admitting a nowhere-zero bidirected flow. Then (1) (Bouchet [7]) G admits a nowhere-zero bidirected 216-flow; 
(2) (Zyka [56], or see [28]) G admits a nowhere-zero bidirected 30-flow;

(3) (Khelladi [28]) $G$ admits a nowhere-zero bidirected 18-flow if $G$ is 4-connected.

Bidirected flow is a generalization of the concept of integer flow. This is because a directed graph $G$ can be considered as a bidirected graph $G^{*}$ with $O\left(G^{*}\right)=0$. However, bidirected flows and integer flows can be quite different due to the existence of those in-edges and out-edges. Some results for integer flows can be generalized to bidirected flows while some other results cannot. The following observation for bidirected 2-flows is a generalization of Tutte's 2-flow characterization. Though the proof for integer 2-flows is very straightforward, the corresponding result for bidirected 2-flow does need a few more steps in the proof.

Proposition 5.1.4 Let $G$ be a connected bidirected graph. Then $G$ admits a nowhere-zero bidirected 2-flow if and only if $G$ is an eulerian graph and $O(G)$ is even.

Integer flows and modular flows are proved to be equivalent for general graphs (see $[45,46]$ or [53]). However, for bidirected graphs, they are not equivalent to each other in many cases. The followings are some examples that a nowhere-zero modular 2-flow in Example 1 and a nowhere-zero modular 3-flow in Example 2 cannot be converted to a nowhere-zero bidirected 2-flow (or 3-flow). For nowhere-zero bidirected modular 3-flows, we are able to establish their equivalent relation for 2-edge connected bidirected graphs.

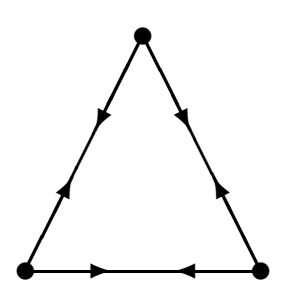

Example 1

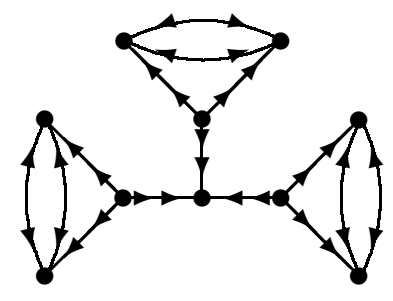

Example 2

The following is one of our main results in this chapter which provides a major step in the proof of Theorem 5.1.6. 
Theorem 5.1.5 Let $G$ be a 2-edge connected bidirected graph. Then $G$ admits a nowherezero bidirected 3-flow if and only if $G$ admits a nowhere-zero modular bidirected 3-flow.

By applying Theorem 5.1.5, we are able to verify Conjecture 5.1.2 for 6-edge connected bidirected graphs.

Theorem 5.1.6 Let $G$ be a 6-edge connected bidirected graph. If $G$ admits a nowhere-zero bidirected flow, then it admits a nowhere-zero bidirected 6 -flow.

\subsection{Proof of Proposition 5.1.4 and its applications}

A circuit is a connected 2-regular graph and a cycle is a graph such that every vertex is of even degree. Let $G$ be a bidirected graph. A circuit $C$ of $G$ is said to be balanced provided that $O(C)$ is even and unbalanced otherwise. A cycle $P$ of $G$ is said to be balanced provided that for each of its component $P^{\prime}, O\left(P^{\prime}\right)$ is even. A collection of cycles $F=\left\{P_{1}, P_{2}, \ldots P_{r}\right\}$ of $G$ is called a proper r-cycle cover of $G$ if every $P_{i}$ is balanced and $\bigcup_{i=1}^{r} E\left(P_{i}\right)=E(G)$. Proof of Proposition 5.1.4.

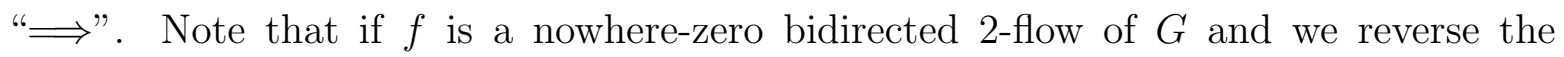
orientation of some edge $e_{1}$, then the resulting graph $G_{1}$ admits a nowhere-zero bidirected 2-flow $f_{1}$ such that $f_{1}(e)=f(e)$ for any $e \neq e_{1}$ and $f_{1}\left(e_{1}\right)=-f\left(e_{1}\right)$. Also, $O(G)=O\left(G_{1}\right)$.

So we may assume that $G$ admits a nowhere-zero bidirected 2-flow $f$ such that $f(e)=1$ for every edge $e \in E(G)$. Therefore, for any $v \in V(G), N^{+}(v)=N^{-}(v)$ which implies $G$ is an eulerian graph. Since $\sum_{v \in V(G)} N^{+}(v)=\sum_{v \in V(G)} N^{-}(v)$ and each ordinary edge contributes 1 to both $\sum_{v \in V(G)} N^{+}(v)$ and $\sum_{v \in V(G)} N^{-}(v)$, the total contribution of non-ordinary edges to $\sum_{v \in V(G)} N^{+}(v)$ must be the same as that to $\sum_{v \in V(G)} N^{-}(v)$. Therefore, we get $\left|E_{i}(G)\right|=\left|E_{o}(G)\right|$ and consequently, $O(G)$ is even. 
"£". Since $G$ is an eulerian graph, let $F=\left\{C_{1}, C_{2}, \ldots, C_{k}\right\}$ be a circuit decomposition of $E(G)$. We will prove it by induction on $k$.

If $k=1$, clearly, it is true. For $k \geq 2$, since $G$ is connected, then there exists $C_{i}$ such that the induced subgraph $G^{*}=G\left[E(G) \backslash E\left(C_{i}\right)\right]$ is connected. Note that $O\left(C_{i}\right)$ is even if and only if $O\left(G^{*}\right)$ is even.

Assume that $O\left(C_{i}\right)$ is even. By induction hypothesis, both $C_{i}$ and $G^{*}$ admits nowherezero bidirected 2-flows, therefore $G$ admits a nowhere-zero bidirected 2-flow. So, we may assume that $O\left(C_{i}\right)$ is odd $\left(O\left(G^{*}\right)\right.$ is odd as well). Let $v$ be any vertex in $V\left(C_{i}\right) \cap V\left(G^{*}\right)$. Suppose $v \in C_{j}$ for some $j \neq i$. Let $C_{i}^{\prime}$ be the new circuit obtained from $C_{i}$ by splitting an edge $e_{i}=v v_{i}$ away from $v$ (becomes $e_{i}=v_{i}^{\prime} v_{i}$, where $v_{i}^{\prime}$ is a new vertex) and adding a new in-edge between $v$ and $v_{i}^{\prime}$ (this operation is called "expending $v$ in $C_{i}$ to an in-edge $e_{i}=v v_{i}^{\prime}$ ). Similarly, we get a new graph $G^{\prime}$ from $G^{*}$ by expending $v$ only in $C_{j}$ to an out-edge $e_{j}=v v_{j}^{\prime}$. Clearly, both $O\left(C_{i}^{\prime}\right)$ and $O\left(G^{\prime}\right)$ are even. By induction hypothesis, $C_{i}^{\prime}$ admits a nowhere-zero bidirected 2-flow $f_{1}$ and $G^{\prime}$ admits a nowhere-zero bidirected 2 -flow $f_{2}$. Note that $-f_{1}$ is also a nowhere-zero bidirected 2-flow of $C_{i}^{\prime}$. So, we can assume $f_{1}\left(e_{i}\right)=f_{2}\left(e_{j}\right)$ (otherwise since $-f_{1}\left(e_{i}\right)=f_{2}\left(e_{j}\right)$ we will use $-f_{1}$ instead of $\left.f_{1}\right)$. Therefore let us define

$$
f(e)= \begin{cases}f_{1}(e) & \text { if } e \in E\left(C_{i}\right) \\ f_{2}(e) & \text { if } e \in E(G) \backslash E\left(C_{i}\right)\end{cases}
$$

Clearly, $f$ is a nowhere-zero bidirected 2-flow of $G$.

The following result is a generalization of a theorem about integer flows.

Proposition 5.2.1 Let $G$ be a bidirected graph. If $G$ has a proper $r$-cycle cover, then $G$ admits a nowhere-zero bidirected $2^{r}$-flow.

Proof. Let $F=\left\{P_{1}, P_{2}, \ldots P_{r}\right\}$ be a proper $r$-cycle cover of $G$. By Proposition 5.1.4, each $P_{i}$ admits a nowhere-zero bidirected 2-flow $f_{i}$. It is easy to verify that $\sum_{i=1}^{r} 2^{r-1} f_{i}$ is a nowhere-zero bidirected $2^{r}$-flow. 


\subsection{Proof of Theorem 5.1.5}

Let us first introduce a useful lemma whose proof is very straightforward.

Lemma 5.3.1 Let $G$ be a bidirected graph, $E_{0}$ be a subset of $E$ and $G_{E_{0}}$ be the bidirected graph obtained from $G$ by reversing the orientation of each edge in $E_{0}$. Then we have

(1) $G$ admits a bidirected $k$-flow if and only if $G_{E_{0}}$ admits a bidirected $k$-flow with the same support.

(2) $G$ admits a bidirected modular $k$-flow if and only if $G_{E_{0}}$ admits a bidirected modular $k$-flow with the same support.

Proof. (1) Suppose that $f$ is a bidirected $k$-flow of $G$. Let

$$
f^{\prime}(e)= \begin{cases}f(e) & \text { if } e \notin E_{0}, \\ -f(e) & \text { if } e \in E_{0}\end{cases}
$$

Clearly $f^{\prime}$ is a bidirected $k$-flow of $G_{E_{0}}$ with the same support.

(2) Suppose that $f$ is a bidirected modular $k$-flow of $G$. Let

$$
f^{\prime}(e)= \begin{cases}k-f(e) & \text { if } e \in E_{0} \text { and } f(e) \neq 0 \\ f(e) & \text { otherwise }\end{cases}
$$

Clearly $f^{\prime}$ is a bidirected modular $k$-flow of $G_{E_{0}}$ with the same support.

Lemma 5.3.2 Let $G$ be a bidirected cubic graph. Then $G$ admits a nowhere-zero bidirected 3-flow if and only if $G$ admits a nowhere-zero bidirected modular 3-flow and $G$ has a perfect matching.

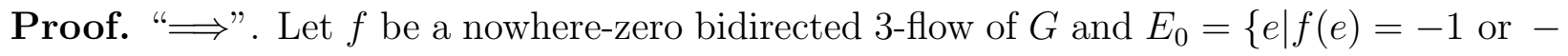
2\}. By the proof of Lemma 5.3.1-(1), $G_{E_{0}}$ admits a positive bidirected 3-flow $f^{\prime}$. Clearly, $f^{\prime}$ 
is also a nowhere-zero bidirected modular 3-flow of $G_{E_{0}}$. By Lemma 5.3.1-(2), $G$ admits a nowhere-zero bidirected modular 3-flow. Because for every vertex $v \in V(G)$, there is exactly one incident edge $e$ such that $f^{\prime}(e)=2$, then $E^{\prime}=\left\{e \mid f^{\prime}(e)=2\right\}$ is a perfect matching of $G$.

"£". Let $f$ be a nowhere-zero bidirected modular 3-flow of $G$ and $E_{0}=\{e \mid f(e)=2\}$. By Lemma 5.3.1-(2), $G^{*}=G_{E_{0}}$ admits a nowhere-zero bidirected modular 3-flow $f^{\prime}$ such that $f^{\prime}(e)=1$ for each edge $e$. Since $G^{*}$ is a cubic graph, then for any vertex $v$ either $E(v)=E^{+}(v)$ or $E(v)=E^{-}(v)$. Let $M$ be a perfect matching of $G^{*}$. Then reverse the direction of each edge $e$ of $M$ and change the value $f^{\prime}(e)$ to be 2 . The resulting nowhere-zero bidirected modular 3-flow is also a nowhere-zero bidirected 3-flow of $G_{M}^{*}$. By Lemma 5.3.1(1), $G^{*}$, and therefore $G$ admit nowhere-zero bidirected 3-flows.

Definition 5.3.3 Let $G$ be a graph and $v$ be a vertex of $G$. Suppose that $F \subset E(v)$, then $G_{[v ; F]}$ is the graph obtained from $G$ by splitting the edges of $F$ away from $v$, that is, adding a new vertex $v^{\prime}$ and change the end $v$ of the edges in $F$ to be $v^{\prime}$.

Lemma 5.3.4 (Nash-Williams [36]) Let $k$ be an even integer and $G$ be a $k$-edge connected graph and $v \in V(G)$. Let a be an integer such that $k \leq a$ and $k \leq d_{G}(v)-a$. Then there is an edge set $F \subset E(v)$ such that $|F|=a$ and $G_{[v ; F]}$ is $k$-edge connected.

Lemma 5.3.5 (Fleischner [14]) Let $G$ be a 2-edge connected graph. Suppose that $v$ is a vertex of $G$ with $d_{G}(v) \geq 4$ and let $e_{0}, e_{1}, e_{2} \in E(v)$. Then either $G_{\left[v ;\left\{e_{0}, e_{1}\right\}\right]}$ or $G_{\left[v ;\left\{e_{0}, e_{2}\right\}\right]}$ is 2-edge connected or $G_{\left[v ;\left\{e_{0}, e_{1}, e_{2}\right\}\right]}$ has more components than $G$.

Lemma 5.3.6 Let $G$ be a 2-edge connected graph. Suppose that $v$ is vertex of $G$ with $d_{G}(v) \geq$ 4 and let $e_{0}, e_{1}, e_{2}, e_{3} \in E(v)$. Then

(1) at least one of $G_{\left[v ;\left\{e_{0}, e_{i}\right\}\right]}(i=1,2,3)$ is 2-edge connected;

(2) in the case of $d_{G}(v)=4$, at least one of $G_{\left[v ;\left\{e_{0}, e_{i}\right\}\right]}(i=1,2)$ is 2-edge connected. 
Proof. By Lemma 5.3.5, it suffices to show that at least one of $G_{\left[v ;\left\{e_{0}, e_{1}, e_{2}\right\}\right]}$ and $G_{\left[v ;\left\{e_{0}, e_{1}, e_{3}\right\}\right]}$ is connected. For convenience, let $e_{i}=v v_{i}(i=0,1,2,3)$. If $d_{G}(v)=4$, then $G_{\left[v ;\left\{e_{0}, e_{1}, e_{2}\right\}\right]}$ is connected. Otherwise $e_{3}$ will be an edge cut of $G$ which is impossible. So, we may assume that $d_{G}(v) \geq 5$. Suppose that $G_{\left[v ;\left\{e_{0}, e_{1}, e_{2}\right\}\right]}$ is disconnected. Since $G$ is 2-edge connected then there is a path $P$ in $G_{\left[v ;\left\{e_{0}, e_{1}, e_{2}\right\}\right]}$ which connects $v_{2}$ to $v_{0}$ without using $e_{2}$. Therefore $G_{\left[v ;\left\{e_{0}, e_{1}, e_{3}\right\}\right]}$ is connected.

Now we are ready to prove Theorem 5.1.5.

\section{Proof of Theorem 5.1.5.}

" $\Longrightarrow$ ". Suppose that $G$ admits a nowhere-zero bidirected 3-flow. Similar to the proof of the first part of Lemma 5.3.2, we can get a nowhere-zero bidirected modular 3-flow of $G$.

"£". Prove by contradiction. Let $G$ be a smallest counterexample with respect to $|E(G)|$. By Lemma 5.3.1, we may assume that $G$ admits a nowhere-zero bidirected modular 3-flow $f$ such that $f(e)=1$ for each edge $e$. Then for any vertex $v$ with $d_{G}(v)=3$ we have either $E(v)=E^{+}(v)$ or $E(v)=E^{-}(v)$.

Claim 1. $\delta(G) \geq 3$.

Otherwise, suppose that there exists $v \in V(G)$ such that $N_{G}(v)=\left\{v_{1}, v_{2}\right\}$. Since $f$ is a nowhere-zero bidirected modular 3-flow of $G$ and $f\left(v_{1} v\right)=f\left(v v_{2}\right)=1$, we can delete the edges $v_{1} v, v v_{2}$ from $G$ and add a new edge $v_{1} v_{2}$ (or a parallel edge if $v_{1} v_{2}$ exists). If $v_{1} v \in E^{+}\left(v_{1}\right)\left(E^{-}\left(v_{1}\right)\right)$, then orient this new edge such that this $v_{1} v_{2} \in E^{+}\left(v_{1}\right)\left(E^{-}\left(v_{1}\right)\right)$; If $v_{2} v \in E^{+}\left(v_{2}\right)\left(E^{-}\left(v_{2}\right)\right)$, then orient this new edge such that this $v_{1} v_{2} \in E^{+}\left(v_{2}\right)\left(E^{-}\left(v_{2}\right)\right)$. The resulting bidirected graph is denoted by $G_{0}$. Then $G_{0}$ is 2-edge connected and admits a nowhere-zero bidirected modular 3-flow with $\left|E\left(G_{0}\right)\right|<|E(G)|$. By the choice of $G, G_{0}$ admits a nowhere-zero bidirected 3-flow. Clearly, from this flow we can easily get a nowherezero bidirected 3-flow of $G$, a contradiction.

Claim 2. There is no $v \in V(G)$ such that $E(v) \cap E^{+}(v) \neq \emptyset$ and $E(v) \cap E^{-}(v) \neq \emptyset$. 
Assume that there exists a vertex $v \in V(G)$ such that $E(v) \cap E^{+}(v) \neq \emptyset$ and $E(v) \cap$ $E^{-}(v) \neq \emptyset$. Then $d_{G}(v) \geq 4$ since, for any degree 3 vertex, either $E(v)=E^{+}(v)$ or $E(v)=E^{-}(v)$.

If $\left|E^{-}(v)\right|$ or $\left|E^{+}(v)\right| \geq 3$, then we may apply Lemma 5.3.6-(1) to split two edges $e^{\prime}, e^{\prime \prime}$ away from the vertex $v$ where $e^{\prime} \in E^{+}(v)$ and $e^{\prime \prime} \in E^{-}(v)$, and the resulting graph is still 2-edge connected and $f$ remains as a nowhere-zero bidirected modular 3-flow. Clearly, this new graph has a degree two vertex and if this graph admits a nowhere-zero 3-flow, then so does $G$. Similar to the proof of Claim 1, we can get a contradiction.

If $0<\left|E^{-}(v)\right|,\left|E^{+}(v)\right| \leq 2$, then $d_{G}(v)=4$ since $\left|E^{+}(v)\right| \equiv\left|E^{-}(v)\right|(\bmod (3))$. By Lemma 5.3.6-(2) and with the same argument as Case 1, we get a contradiction as well.

By Claim 1 and Claim 2, we have either $E(v)=E^{+}(v)$ or $E(v)=E^{-}(v)$ for any $v \in$ $V(G)$. Therefore, $d_{G}(v) \equiv 0(\bmod 3)$ for any $v \in V(G)$. For any $v$ with $d_{G}(v) \geq 6$, we may apply Lemma 5.3.4 to split this vertex into several degree 3 vertices and the resulting graph is still 2-edge connected and $f$ remains as a nowhere-zero bidirected modular 3-flow. Also if the resulting graph admits a nowhere-zero bidirected 3-flow, then so does $G$. Recursively splitting high degree vertices, we obtain a 2-edge connected cubic graph $G^{*}$ which admits a nowhere-zero bidirected modular 3-flow.

By Petersen Theorem [38], the 2-connected, cubic graph $G^{*}$ has a perfect matching. Then by Lemma 5.3.2, $G^{*}$ admits a nowhere-zero bidirected 3-flow. So, from this flow of $G^{*}$, we can get a nowhere-zero bidirected 3-flow of $G$, a contradiction.

\subsection{Proof of Theorem 5.1.6}

Product of flows is a method used in the proof of the theorems of 8-flow [23], 4-flow [24] and 6-flow [41]. The following lemma generalizes this method for bidirected flows of graphs and is to be used in the proof of Theorem 5.1.6. 
Lemma 5.4.1 Let $G$ be a bidirected graph. If $G$ admits a bidirected $k_{1}$-flow $f_{1}$ and a bidirected $k_{2}$-flow $f_{2}$ such that $\operatorname{supp}\left(f_{1}\right) \cup \operatorname{supp}\left(f_{2}\right)=E(G)$, then $G$ admits a nowhere-zero bidirected $k_{1} k_{2}$-flow.

Proof. Let $f(e)=f_{1}(e)+k_{1} f_{2}(e)$ for every $e \in E(G)$, then it is easy to verify that $f$ is a nowhere-zero bidirected $k_{1} k_{2}$-flow.

Let $G$ be a bidirected graph. A subgraph $C$ of $G$ is called a bidirected circuit of $G$ provided that either $C$ is a balanced circuit of $G$ or $C$ is the union of two unbalanced circuits sharing exactly one vertex or $C$ is the union of two vertex-disjoint unbalanced circuits and a simple path meeting each of the two circuits at exactly one of its end points.

We extend Seymour's closure operation [41] to bidirected graphs as follows:

For a positive integer $k$, if $X$ is a subgraph of $G$, then the $k$-closure of $X$ in $G$, denoted by $\langle X\rangle_{k}$, is the (unique) maximal subgraph of $G$ of the form $X \cup C_{1} \cup, \ldots \cup C_{n}$, where for every $i, 1 \leq i \leq n, C_{i}$ is a bidirected circuit of $G$ and $\left|E\left(C_{i}\right) \backslash E\left(X \cup C_{1} \cup, \ldots \cup C_{i-1}\right)\right| \leq k$.

By the Theorem 4.3 in [28], we have the following:

Lemma 5.4.2 Let $G$ be a connected bidirected graph which admits a nowhere-zero bidirected flow. Let $E^{\prime}$ be a subset of $E(G)$ such that the induced graph $G\left[E^{\prime}\right]$ is connected and spanning. Then $\left\langle E^{\prime}\right\rangle_{2}=E(G)$.

By the Proposition 2.2 in [28], we have the following:

Lemma 5.4.3 Let $G$ be a bidirected graph and $k \geq 3$ be a prime integer. Let $E^{\prime}$ be a subset of $E(G)$ such that $\left\langle E^{\prime}\right\rangle_{k-1}=E(G)$. Then $G$ admits a bidirected integer flow $f$ such that $f(e) \not \equiv 0(\bmod k)$ for every element e of $E(G) \backslash E^{\prime}$.

The bidirected flow $f$ obtained in Lemma 5.4 .3 can be considered as a bidirected modular $k$-flow with the same support though the value of $f$ can be very large. 
Definition 5.4.4 Let $H_{1}$ and $H_{2}$ be two subgraphs of a graph $G$. The symmetric difference of $H_{1}$ and $H_{2}$, denoted by $H_{1} \triangle H_{2}$, is the subgraph of $G$ induced by the set of edges $\left[E\left(H_{1}\right) \cup E\left(H_{2}\right)\right] \backslash\left[E\left(H_{1}\right) \cap E\left(H_{2}\right)\right]$. The symmetric difference of finitely many subgraphs $\left\{H_{1}, \cdots, H_{t}\right\}$ of $G$ is defined recursively as

$$
\triangle_{1 \leq i \leq t} H_{i}=H_{1} \triangle \cdots \triangle H_{t-1} \triangle H_{t}=\left[H_{1} \triangle \cdots \triangle H_{t-1}\right] \triangle H_{t} .
$$

The following is a well-known fact:

Lemma 5.4.5 Let $\left\{H_{1}, \cdots, H_{t}\right\}$ be a family of subgraphs of $G$. Let $S=H_{1} \triangle \cdots \triangle H_{t}$. Then

(1) $S$ is the subgraph of $G$ induced by the edges contained in an odd number of $H_{i}^{\prime} s$;

(2) If $H_{1}, \cdots, H_{t}$ are all cycles of $G$, then $S$ is also a cycle.

Now we are ready to prove Theorem 5.1.6.

\section{Proof of Theorem 5.1.6.}

Since $G$ is 6-edge connected, by Lemma 4.2.2, $G$ has three edge-disjoint spanning trees $T_{1}, T_{2}$ and $T_{3}$. Because $G$ admits a nowhere-zero bidirected flow, by Lemma $5.4 .2,\left\langle T_{i}\right\rangle_{2}=$ $E(G)$ for $1 \leq i \leq 3$.

For $1 \leq i \leq 3$, let $k=3, E^{\prime}=E\left(T_{i}\right)$ and apply Lemma 5.4.3, we get a bidirected integer flow $f_{i}$ of $G$ such that $f_{i}(e) \not \equiv 0(\bmod 3)$ for every element $e$ of $E(G) \backslash E\left(T_{i}\right)$. Clearly $\operatorname{supp}\left(f_{1}\right) \supseteq E(G) \backslash E\left(T_{1}\right)$. Since $G\left[\operatorname{supp}\left(f_{1}\right)\right]$ contains two edge-disjoint spanning trees $T_{2}$ and $T_{3}$, the subgraph $G\left[\operatorname{supp}\left(f_{1}\right)\right]$ is 2-edge connected and spanning. Similarly, $\operatorname{supp}\left(f_{2}\right) \supseteq E(G) \backslash E\left(T_{2}\right), \operatorname{supp}\left(f_{3}\right) \supseteq E(G) \backslash E\left(T_{3}\right)$ and both $G\left[\operatorname{supp}\left(f_{2}\right)\right]$ and $G\left[\operatorname{suppt}\left(f_{3}\right)\right]$ are 2-edge connected and spanning. From each $f_{i}(1 \leq i \leq 3)$, we can get a bidirected modular 3 -flow $f_{i}^{\prime}$ such that $\operatorname{supp}\left(f_{i}\right)=\operatorname{supp}\left(f_{i}^{\prime}\right)$. Then, by Theorem 5.1.5, we get a bidirected 3-flow $f_{i}^{\prime \prime}$ such that $\operatorname{supp}\left(f_{i}^{\prime \prime}\right)=\operatorname{supp}\left(f_{i}\right)$ for $i=1,2,3$. 
Let $T_{i}, T_{j}$ be two edge-disjoint spanning trees. For each $e \in T_{j}$, there is a unique circuit $C_{e}$ of $G$ contained in $T_{i} \cup\{e\}$. By Lemma 5.4.5-(2), $C_{i}^{j}=\triangle_{e \in E\left(T_{j}\right)} C_{e}$ is eulerian. By Lemma 5.4.5-(1), $\left(E\left(T_{i}\right) \cup E\left(T_{j}\right)\right) \supseteq E\left(C_{i}^{j}\right) \supseteq E\left(T_{j}\right)$, then $C_{i}^{j}$ is also connected and spanning. Let us consider the following two cases.

Case 1. There exists one $C_{i}^{j}$ such that $O\left(C_{i}^{j}\right)$ is even.

Without loss of generality, we may assume that $O\left(C_{1}^{2}\right)$ is even. Since $C_{1}^{2}$ is eulerian and connected, by Proposition 5.1.4, $C_{1}^{2}$ admits a nowhere-zero bidirected 2-flow. Therefore, $G$ admits a bidirected 2-flow $f_{1,2}$ such that $\operatorname{supp}\left(f_{1,2}\right) \supseteq E\left(T_{2}\right)$. Also $f_{2}^{\prime \prime}$ is bidirected 3-flow such that $\operatorname{supp}\left(f_{2}^{\prime \prime}\right) \supseteq E(G) \backslash E\left(T_{2}\right)$. By Lemma 5.4.1, we get a nowhere-zero bidirected 6-flow of $G$.

Case 2. All $O\left(C_{i}^{j}\right)^{\prime}$ 's are odd for $1 \leq i \neq j \leq 3$.

Since $O\left(C_{1}^{3}\right)$ is odd, the eulerian subgraph $C_{1}^{3}$ contains an unbalanced circuit $c_{1}^{3}$. Clearly, $E\left(c_{1}^{3}\right) \subseteq\left(E\left(T_{1}\right) \cup E\left(T_{3}\right)\right)$. Because $O\left(C_{1}^{2}\right)$ is odd and $E\left(T_{2}\right) \subseteq E\left(C_{1}^{2}\right) \subseteq E\left(T_{1}\right) \cup E\left(T_{2}\right)$, then $C^{*}=C_{1}^{2} \triangle c_{1}^{3}$ is an eulerian, connected and spanning subgraph of $G$ with $O\left(C^{*}\right)$ even. Clearly, $E\left(T_{2}\right) \subseteq E\left(C^{*}\right)$. By Proposition 5.1.4, $C^{*}$ admits a nowhere-zero bidirected 2-flow $f^{*}$ with $\operatorname{supp}\left(f^{*}\right) \supseteq E\left(T_{2}\right)$. Similar to Case 1, by Lemma 5.4.1, we can get a nowhere-zero bidirected 6-flow of $G$. 


\section{Bibliography}

[1] G. Agnarsson, M. M. Halldorsson, Coloring squares of graphs, SIAM Journal of Discrete Math., 16(4) (2003), 651-662

[2] Y. Alavi, G. Chartrand, The existence of 2-factors in squares of graphs, Czechoslovak Math. Journal, 25 (1975), 79-83.

[3] K. Appel, W. Haken, Every map is four colorable, Part I: Discharging, Illinois Journal of Math., 21 (1977), 429-490.

[4] K. Appel, W. Haken and J. Koch, Every map is four colorable, Part II: Reducibility, Illinois Journal of Math., 21 (1977), 491-567.

[5] K. Appel, W. Haken, Every map is four colorable, Contemp. Math. AMS, 98 (1989).

[6] J. A. Bondy, U. S. R. Murty, Graph Theory with Applications. Macmillan, London, (1976).

[7] A. Bouchet, Nowhere-zero integer flows on a bidirected graph, Journal of Combin. Theory Ser. B., 34 (1983), 279-292.

[8] P. A. Catlin, A reduction method to find spanning eulerian subgraph, Journal of Graph Theory, 12 (1988), 29-45.

[9] P. A. Catlin, Double cycle covers and the Petersen graph, Journal of Graph Theory, 13 (1989), 465-483. 
[10] P. A. Catlin, Z.-Y. Han and H.-J. Lai, Graphs without spanning closed trails, Discrete Math., 160 (1996), 81-91.

[11] Z-H Chen, H-J Lai, H-Y Lai, Nowhere zero flows in line graphs, Discrete Math. 230 (2001), 133-141.

[12] M. Devos, R. Xu, and G-X Yu, Nowhere-zero $Z_{3}$-flows through $Z_{3}$-connectivity, submitted to Discrete Math., 2003.

[13] H. Fleischner, The square of every two-connected graph is Hamiltonian, Journal of Combin. Theory Ser. B 16 (1974), 29-34.

[14] H. Fleischner, Eine gemensame Basis für die Theorie der eulerischen Graphen und den satz von Petersen, Monatsh. Math. 81 (1976), 267-278.

[15] A. Galluccio, L. A. Goddyn, The circular flow number of a 6-edge-connected graph is less than four, Combinatorica, 22 (2002), 455-459.

[16] L. A. Goddyn, M. Tarsi and C.-Q. Zhang, On $(k, d)$-colorings and fractional nowhere zero flows, Journal of Graph Theory, 28 (1998), 155-161.

[17] H. Grötzsch, Ein Dreifarbensatz für dreikreisfreie Netze auf der Kugel, Wissenschaftliche Zeitschrift der Martin-Luther-Universität Halle-Wittenberg, Mathematisch-Naturwissenschaftliche Reihe, 8 (1958/1959), 109-120.

[18] D. Gusfield, Connectivity and edge-disjoint spanning trees, Inform. Process. Lett. 16 (1983), 87-89.

[19] A. J. Hoffman, Some recent applications of the theorem of linear inequalities to extremal combinatorial analysis, Proc. Symp. Appl. Math., 10 (1960), 113-127.

[20] W. Imrich and R. Skrekovski, A theorem on integer flows on Cartesian product of graphs, Journal of Graph Theory, 43 (2003), 93-98. 
[21] A. Itai, M. Rodeh, Covering a graph by circuits, in Automata, Languages and Programming, Lecture Notes in Computer Science 62, Springer-Verlag, Berlin, (1978), 289-299.

[22] B. Jackson, On circuit covers, circuit decompositions and Euler tours of graphs. Surveys in combinatorics, 1993 (Keele), London Math. Soc. Lecture Note Ser., 187, Cambridge Univ. Press, Cambridge, (1993), 191-210.

[23] F. Jaeger, Flows and generalized coloring theorems in graphs, Journal of Combin. Theory Ser. B., 26 (1979), 205-216.

[24] F. Jaeger, A note on supereulerian graphs, Journal of Graph Theory, 3 (1979), 91-93.

[25] F. Jaeger, Nowhere-zero flow problems, in: L. Beineke and R. Wilson, eds., Selected Topics in Graph Theory 3 (Wiley, New York, 1988), 71-95.

[26] F. Jaeger, N. Linial, C. Payan and M. Tarsi, Group connectivity of graphs - a nonhomogeneous analogue of nowhere-zero flow properties. Journal of Comb. Theory, Ser. B 56 (1992), 165-182.

[27] I. Kang, Suh-Ryung Kim, Y. Shin and Y. Nam, Graphs satisfying inequality $\theta\left(G^{2}\right) \leq$ $\theta(G)$, Discrete Math., 250 (2002), 259-264.

[28] A. Khelladi, Nowhere-zero integer chains and flows in bidirected graphs, Journal of Combin. Theory Ser. B., 43 (1987), 95-115.

[29] P. A. Kilpatrick, Tutte's first colour-cycle conjecture, Ph. D. Thesis, Cape Town, (1975).

[30] S. Kundu, Bounds on the number of disjoint spanning trees, Journal of Combin. Theory Ser. B., 17, (1974), 199-203.

[31] H.-J. Lai and C.-Q. Zhang, Nowhere-zero 3-flows of highly connected graphs, Discrete Math., 110 (1992), 179-183. 
[32] H-J Lai, Nowhere-zero 3-flows in locally connected graphs. Journal of Graph Theory, 42 (2003), 211-219.

[33] H-J Lai, R. Xu and C-Q Zhang, Note on circular flow numbers of graphs, submitted to Combinatorica, 2004.

[34] H-J Lai, R. Xu and C-Q Zhang, Group connectivity of triangularly connected graphs, in preparation.

[35] C. S. J. A. Nash-Williams, Edge-disjoint spanning trees of finite graphs, Journal of London Math. Soc., 36 (1961), 445-450.

[36] C. ST J. A. Nash-Williams, Connected detachment of graphs and generalized Euler graphs, Journal of London Math. Soc., (1988), 17-29.

[37] L. Nebesk'y, On the existence of 1-factors in partial squares of graphs. Czechoslovak Math. Journal, 29(104)(1979), 349-352

[38] J. Peterson, Die Theorie der regulären graphs, Acta Math., 15 (1891), 193-220.

[39] V. P. Polesskii, A lower bound for the reliability of information network, Prob. Inf. Transmission 7, (1971), 165-171.

[40] G. Schaar, Remarks on Hamiltonian properties of squares of graphs, Arch. Math. (Brno), 25 (1-2) (1989), 61-72.

[41] P. D. Seymour, Nowhere-zero 6-flows, Journal of Combin. Theory Ser. B., 30 (1981), 130-136.

[42] R. Steinberg, Grötzsch's theorem dualized, M. Math Thesis, University of Waterloo, Ontario, Canada, (1976).

[43] R. Steinberg and D. H. Younger, Grötzsch's theorem for the projective plane, Ars Combinatoria, 28, (1989), 15-31. 
[44] B. Toft, T. R. Jensen, Graph coloring problems, John Wiley \& Sons, Inc., New York (1995).

[45] W. T. Tutte, On the embedding of linear graphs in surfaces, Proc. London Math. Soc., Ser. 2, 51 (1949), 474-483.

[46] W. T. Tutte, A contribution on the theory of chromatic polynomial, Canad. Journal of Math., 6 (1954), 80-91.

[47] W. T. Tutte, On the problem of decompositing a graph into $n$ connected factors, Journal of London Math. Soc., 36 (1961), 221-230.

[48] W. T. Tutte, On the algebraic theory of graph colourings, Journal of Combin. Theory, 1 (1966), 15-50.

[49] R. Xu, Note on acyclic colorings of graphs, Ars Combinatoria, accepted September 2002.

[50] R. Xu, C-Q Zhang, Nowhere-zero 3-flows in squares of graphs. Electronic Journal of Combinatorics, 10 (2003), R5.

[51] R. Xu, C-Q Zhang, On flows of bidirected graphs, submitted to Discrete Math., 2003.

[52] D. H. Younger, Interger flows, Journal of Graph Theory, 7 (1983), 349-357.

[53] C. Q. Zhang, Integer Flows and Cycle Covers of Graphs, Marcel Dekker, New York (1997).

[54] C. Q. Zhang, Integer flows and cycle covers, plenary lecture at graph theory workshop, Nanjing Normal University, April, 1998.

[55] C.-Q. Zhang, Circular flows of nearly eulerian graphs and vertex-splitting, Journal of Graph Theory, 40 (2002), 147-161. 
[56] O. Zyka, Nowhere-zero 30-flow on bidirected graphs, Thesis, Charles University, Praha (1987); KAM-DIMATIA Series 87-26. 